\title{
DAMPING EFFECTS IN BOUNDARY LAYERS FOR ROTATING FLUIDS WITH SMALL VISCOSITY
}

\author{
VAN-SANG NGO
}

\begin{abstract}
The goal of this paper is to study the system of rotating fluids between two infinite parallel plates with Dirichlet boundary conditions and with small viscosity which vanishes when the Rossby number goes to zero. We want to improve the convergence result of [16] and show the global in time convergence of the weak solution of the system of rotating fluids towards the solution of a twodimensional damped Euler system with three components, using the decay in time of the $H^{s}$-norm $(s>2)$ of the limiting solution.
\end{abstract}

\section{INTRODUCTION}

In this paper, we consider the system of fast rotating incompressible fluids

$$
\left\{\begin{array}{l}
\partial_{t} u^{\varepsilon}-\nu_{h}(\varepsilon) \Delta_{h} u^{\varepsilon}-\beta \varepsilon \partial_{3}^{2} u^{\varepsilon}+u^{\varepsilon} \cdot \nabla u^{\varepsilon}+\frac{e_{3} \wedge u^{\varepsilon}}{\varepsilon}+\nabla p^{\varepsilon}=0 \\
\operatorname{div} u^{\varepsilon}=0 \\
u^{\varepsilon}{ }_{t=0}=u_{0}^{\varepsilon},
\end{array}\right.
$$

for $t>0$ and for $\left.x \in \Omega=\mathbb{R}^{2} \times\right] 0,1[$, coupled with the Dirichlet boundary conditions

$$
u^{\varepsilon}\left(t, x_{1}, x_{2}, 0\right)=u^{\varepsilon}\left(t, x_{1}, x_{2}, 1\right)=0 .
$$

Here, the fluid rotates around $e_{3}$-axis and $\varepsilon>0$ stands for the Rossby number, which will be small and goes to zero. In this system, the vertical viscosity is $\nu_{v}=\beta \varepsilon$, with $\beta>0$ and the horizontal viscosity $\nu_{h}=\nu_{h}(\varepsilon)>0$ depends on $\varepsilon$ and goes to zero as $\varepsilon$ goes to zero. The classical isotropic diffusion $-\nu \Delta$ is replaced by an anisotropic diffusion of the form

$$
-\nu_{h} \Delta_{h}-\nu_{v} \partial_{3}^{2}=-\nu_{h}(\varepsilon)\left(\partial_{1}^{2}+\partial_{2}^{2}\right)-\beta \varepsilon \partial_{3}^{2},
$$

where $\partial_{i}$ is the partial derivative with respect to the variable $x_{i}$. This anisotropy is often considered in meteorology and oceanography, where vertical viscosity $\nu_{v}$ is taken to be much smaller than the horizontal viscosity $\nu_{h}$. We refer to Pedlosky [25] and Greenspan [15] for more details about physical considerations.

It is clear that the Coriolis force term has no contribution in the energy estimates for the system (1.1). In the case where $\nu_{h}>0$ and $\nu_{v}>0$, independent of $\varepsilon$, the global existence of weak solutions of (1.1) in the sense of Leray can be proved by the same method as in the case of the Navier-Stokes equations (see J. Leray, [19]). By using the same arguments as in H. Fujika and T. Kato [12] for the Navier-Stokes equations, one can show local existence and uniqueness of a strong solution of (1.1), the lifespan of which is bounded from below, uniformly with respect to $\varepsilon$. We also want to refer to [6], [7], [17], [23], [24], $\ldots$, and the references therein for the anisotropic cases where the vertical viscosity is zero. In the case where $\nu_{h}$ and $\nu_{v}$ vanish or depend on $\varepsilon$, using the same method as for the Euler system, we can prove that the system (1.1) is locally well-posed when the initial data $u_{0}^{\varepsilon}$ belong to a smooth enough Sobolev space, independent of $\nu_{h}$ and $\nu_{v}$.

We have not mentioned the role of the Coriolis force yet. Actually in fast rotating systems with small Rossby number, the Coriolis force plays an important role. In the experiment of G.I. Taylor (see [11]), drops of dye injected into a rapidly rotating, homogeneous fluid, within a few rotations, formed perfectly vertical sheets of dyed fluid, known as Taylor curtains. In large-scale atmospheric and oceanic flows, the fluid motions also have a tendency towards columnar behaviors (Taylor columns). For example, currents in the western North Atlantic have been observed to extend vertically over several thousands meters without significant change in amplitude and direction ([26]).

Date: October 15, 2019.

1991 Mathematics Subject Classification. 76D03; 76D05; 76U05.

Key words and phrases. Damping Euler; Navier-Stokes; Rotating fluids; Ekman boundary layer. 
Fast rotating flows have first been considered in periodic domains. In the work of Babin, Mahalov and Nicolaenko [1], [2], the authors studied the isotropic case, where the diffusion term is $-\nu \Delta$, with $\nu>0$ independent of $\varepsilon$. For any initial data in appropriate Sobolev spaces, they proved the global existence and uniqueness of a smooth solution when the rotation is fast enough (that is, the Rossby number $\varepsilon$ is small enough). The same result was proven by Gallagher in [13], using the method of Schochet [27], for fast rotating fluid systems and also for more general parabolic systems. It was also shown in [1], [2] and [13] that, when $\varepsilon$ goes to zero, the fast rotating system converges to a two-dimensional Navier-Stokes system with three components, if $\nu_{h}>0$ is fixed, or to a two-dimensional Euler system with three components, if $\nu_{h}$ is zero or goes to zero as $\varepsilon$ goes to zero. The convergence in the more general case, where the rotation axis is fixed but not the amplitude, was proven in [14] by Gallagher and Saint-Raymond. The anisotropic case, with zero vertical viscosity $\left(\nu_{v}=0\right)$, in periodic domains, that is the system $(1.1)$, with $\nu_{h}>0$ independent of $\varepsilon$, in the torus $\mathbb{T}^{3}$, was studied later by Paicu in [23].

In the case of rotating fluids in the whole space $\mathbb{R}^{3}$, it was proved in [6], [7] and [14] that, if the initial data are divergence-free vector fields belonging to $\mathbf{H}^{0, s}\left(\mathbb{R}^{3}\right)$ then, when $\varepsilon$ goes to zero, the limiting system corresponding to (1.1) is zero. We remark that in this paper, in order to simplify the notations, we use the bold character $\mathbf{X}$ to indicate the space of vector fields, each component of which belongs to the space $X$. The main idea consists in proving and using Strichartz estimates for the associated linear system, which shows that, in the case of $\mathbb{R}^{3}$, the free oscillation waves of the fluid propagate to infinity and the energy of the fluid decreases. Using this property, it was also proved in [7] that the system (1.1), with $\nu_{h}>0$ fixed, has a unique, global solution when the rotation is fast enough. The same result in the case where $\nu_{h}$ slowly goes to zero as $\varepsilon \rightarrow 0$, say $\nu_{h}=\varepsilon^{\alpha}$, with $\left.\left.\alpha \in\right] 0, \alpha_{0}\right]$, for a certain $\alpha_{0}>0$, was proved in [22]. If the initial data is not in $\mathbf{H}^{0, s}\left(\mathbb{R}^{3}\right)$ but is the sum of a $2 \mathrm{D}$ part in $L^{2}\left(\mathbb{R}_{h}^{2}\right)^{3}$ and a $3 \mathrm{D}$ part in $\mathbf{H}^{0, s}\left(\mathbb{R}^{3}\right), s>\frac{1}{2}$, then the limit system is not zero but a $2 \mathrm{D}$ Navier-Stokes system $\left(\nu_{h}>0\right.$ fixe or a $2 \mathrm{D}$ Euler system $\left(\nu_{h} \rightarrow 0\right)$ with three components, as $\varepsilon$ goes to zero. Using the global wellposedness of the limit system, the global existence of a strong solution when $\varepsilon$ is small enough can be proved in the case of fixed $\nu_{h}>0$ (see [7]).

The case of a domain between two parallel plates (with Dirichlet boundary conditions) is very different from the case of a domain without boundary $\left(\mathbb{R}^{3}\right.$ or $\left.\mathbb{T}^{3}\right)$ mentioned above. Indeed, when the rotation goes to infinity $(\varepsilon \rightarrow 0)$, the fluid has the tendency to have a columnar behavior (the Taylor-Proudman theorem). However, the Taylor columns are only formed in the interior of the domain. Near the boundary, the Taylor columns are destroyed and very thin boundary layers are formed (Ekman boundary layers). Inside the boundary layers, the behavior of the fluid becomes very complex and the friction slows the fluid down in a way that the velocity is zero on the boundary. As a consequence, in the limiting system, an additional damping term of the form $\gamma \bar{u}, \gamma>0$, appears. The coefficient $\gamma$ was proved to be $\sqrt{2 \beta}$ in [16] (we will explain the calculation of this coefficient in the appendix). This phenomenon is well known in fluid mechanics as the Ekman pumping.

For any $\varepsilon>0$, and for any $u_{0}^{\varepsilon} \in \mathbf{L}^{2}(\Omega)$, the system (1.1) possesses a weak Leray solution

$$
u^{\varepsilon} \in \mathbf{L}^{\infty}\left(\mathbb{R}_{+}, \mathbf{L}^{2}(\Omega)\right) \cap \mathbf{L}^{2}\left(\mathbb{R}_{+}, \dot{\mathbf{H}}^{1}(\Omega)\right) .
$$

If $\nu_{h}>0$ is fixed, then taking into account the Ekman pumping, the limiting system is the following 2D damped Navier-Stokes sytem

$$
\left\{\begin{array}{l}
\partial_{t} \bar{u}^{h}-\nu_{h} \Delta_{h} \bar{u}^{h}+\bar{u}^{h} \cdot \nabla_{h} \bar{u}^{h}+\sqrt{2 \beta} \bar{u}^{h}=-\nabla_{h} \bar{p} \\
\partial_{3} \bar{u}=0, \operatorname{div}_{h} \bar{u}^{h}=0, \bar{u}^{3}=0 \\
\bar{u}_{\left.\right|_{t=0}}=\bar{u}_{0}=\left(\bar{u}_{0}^{1}, \bar{u}_{0}^{2}, 0\right) .
\end{array}\right.
$$

When $\varepsilon \rightarrow 0$, $u^{\varepsilon}$ was proved to converge to the solution of the limiting system in $\mathbf{L}^{\infty}\left(\mathbb{R}_{+}, \mathbf{L}^{2}(\Omega)\right)$, for $\Omega=\mathbb{T}_{h}^{2} \times[0,1]$, by Grenier and Masmoudi [16] and by Masmoudi [20] in the case where the initial data are well prepared (i.e. $\lim _{\varepsilon \rightarrow 0} u_{0}^{\varepsilon}=\bar{u}_{0}=\left(\bar{u}_{0}^{1}\left(x_{1}, x_{2}\right), \bar{u}_{0}^{2}\left(x_{1}, x_{2}\right), 0\right)$ in $\mathbf{L}^{2}\left(\mathbb{T}_{h}^{2} \times[0,1]\right)$ and by Masmoudi [21] in the case of general initial data. The same result for $\Omega=\mathbb{R}_{h}^{2} \times[0,1]$ was prove by Chemin et al. in [8], using Strichartz estimates.

If $\nu_{h}=\nu_{h}(\varepsilon) \rightarrow 0$ when $\varepsilon \rightarrow 0$, the limiting system is now the following 2D damped Euler system

$$
\left\{\begin{array}{l}
\partial_{t} \bar{u}^{h}+\bar{u}^{h} \cdot \nabla_{h} \bar{u}^{h}+\sqrt{2 \beta} \bar{u}^{h}=-\nabla_{h} \bar{p} \\
\partial_{3} \bar{u}=0, \operatorname{div}_{h} \bar{u}^{h}=0, \bar{u}^{3}=0 \\
\bar{u}_{\left.\right|_{t=0}}=\bar{u}_{0}=\left(\bar{u}_{0}^{1}, \bar{u}_{0}^{2}, 0\right) .
\end{array}\right.
$$


In [16], [20], [21] (for $\Omega=\mathbb{T}_{h}^{2} \times[0,1]$ ) and in [8] (for $\Omega=\mathbb{R}_{h}^{2} \times[0,1]$ ), the solution $u^{\varepsilon}$ can still be proved to converge to the solution of (1.3) in $\mathbf{L}_{\text {loc }}^{\infty}\left(\mathbb{R}_{+}, \mathbf{L}^{2}(\Omega)\right)$. The local convergence with respect to the time variable is due to the local in time boundedness of the $\mathbf{H}^{\sigma}\left(\mathbb{R}_{h}^{2}\right)$-norm $(\sigma>2)$ of the solution of $(1.3)$ used in the previous results.

The goal of this paper is to improve the above results and to prove that, in the case where $\nu_{h}=\nu_{h}(\varepsilon)$ goes to zero as $\varepsilon$ goes to zero, the convergence of $u^{\varepsilon}$ towards $\bar{u}$ is global in time. We consider the system (1.1) in the domain $\Omega=\mathbb{R}_{h}^{2} \times[0,1]$ (the same result can be proved for the domain $\Omega=\mathbb{T}_{h}^{2} \times[0,1]$ with minor changes) with Dirichlet boundary conditions and with the well-prepared data as in [16] or [20]. The main result of the paper is the following theorem.

Theorem 1.1. We suppose that $\nu_{h}(\varepsilon) \rightarrow 0$ as $\varepsilon \rightarrow 0$. Let $u_{0}^{\varepsilon} \in \mathbf{L}^{2}\left(\mathbb{R}_{h}^{2} \times[0,1]\right)$ be a family of initial data such that

$$
\lim _{\varepsilon \rightarrow 0} u_{0}^{\varepsilon}=\bar{u}_{0}=\left(\bar{u}_{0}^{1}\left(x_{1}, x_{2}\right), \bar{u}_{0}^{2}\left(x_{1}, x_{2}\right), 0\right) \quad \text { in } \quad \mathbf{L}^{2}\left(\mathbb{R}_{h}^{2} \times[0,1]\right),
$$

where $\bar{u}_{0}$ is a divergence-free two-dimensional vector field in $\mathbf{H}^{\sigma}\left(\mathbb{R}_{h}^{2}\right), \sigma>2$. Let $\bar{u}$ be the solution of the limiting system (1.3) with initial data $\bar{u}_{0}$ and, for each $\varepsilon>0$, let $u^{\varepsilon}$ be a weak solution of (1.1) with initial data $u_{0}^{\varepsilon}$. There exists a positive constant $C=C\left(\bar{u}_{0}\right)>0$ such that, if $\nu_{h}(\varepsilon) \geq C\left(\bar{u}_{0}\right) \varepsilon$, then

$$
\lim _{\varepsilon \rightarrow 0}\left\|u^{\varepsilon}-\bar{u}\right\|_{\mathbf{L}^{\infty}\left(\mathbb{R}_{+}, \mathbf{L}^{2}\left(\mathbb{R}_{h}^{2} \times[0,1]\right)\right)}=0 .
$$

The key of the proof of Theorem 1.1 consists in the careful study of the limiting system (1.3). We prove that the damping term $\sqrt{2 \beta} \bar{u}$ in the system (1.3) plays a very important role which allow us to prove the exponential decay in time of the $\mathbf{H}^{\sigma}\left(\mathbb{R}_{h}^{2}\right)$-norm, $\sigma>2$, of the solution. More precisely, in Section 3 , we prove the following theorem.

Theorem 1.2. Let $\bar{u}_{0}=\left(\bar{u}_{0}^{1}\left(x_{1}, x_{2}\right), \bar{u}_{0}^{2}\left(x_{1}, x_{2}\right), 0\right) \in \mathbf{L}^{2}\left(\mathbb{R}_{h}^{2}\right)$ be a divergence-free vector field, the horizontal vorticity of which $\bar{w}_{0}=\partial_{1} \bar{u}_{0}^{2}-\partial_{2} \bar{u}_{0}^{1} \in L^{2}\left(\mathbb{R}_{h}^{2}\right) \cap L^{\infty}\left(\mathbb{R}_{h}^{2}\right)$. Then, the system (1.3), with initial data $\bar{u}_{0}$, has a unique, global solution

$$
\bar{u} \in \mathbf{C}\left(\mathbb{R}_{+}, \mathbf{L}^{2}\left(\mathbb{R}_{h}^{2}\right)\right) \cap \mathbf{L}^{\infty}\left(\mathbb{R}_{+}, \mathbf{L}^{2}\left(\mathbb{R}_{h}^{2}\right)\right) .
$$

Moreover, if $\bar{u}_{0}$ belongs to $\mathbf{H}^{\sigma}\left(\mathbb{R}_{h}^{2}\right), \sigma>2$, then there exists a positive constant $C$ depending on $\beta$ and $\left\|\bar{u}_{0}\right\|_{\mathbf{H}^{\sigma}\left(\mathbb{R}_{h}^{2}\right)}$ such that, for any $t>0$,

$$
\|\bar{u}(t)\|_{\mathbf{H}^{\sigma}\left(\mathbb{R}_{h}^{2}\right)} \leq C e^{-t \sqrt{2 \beta}}
$$

We note that a unique global solution of (1.3) exists thanks to the Yudovitch theorem [28] and the exponential decay is not true in the case of the two-dimensional non-damped Euler system, where the $\mathbf{L}^{\infty}$-norm of the gradient of the vorticity may have a double exponential growth in time (see Bahouri \& Chemin [3], Kiselev \& Šverák [18] and the references therein). The goal of Section 3 is to prove the exponential decay properties of this solution in the case of damped Euler system.

The paper is organized as follows. In the next section, we briefly recall the dyadic decompositions and the Littlewood-Paley theory which will be used in Section 3 to prove Theorem 1.2. In the last section, we prove the main theorem (Theorem 1.1) on the convergence of weak solutions of the system (1.1) towards the solution of the system (1.3). Finally, for the convenience of the reader, we will very briefly recall the construction of Ekman boundary layers and the formation of the limiting system in the appendix. We will also explain why the damping term appears in the limiting system (the Ekman pumping).

\section{BRIEF RECALL OF DYADIC DECOMPOSITIONS}

In this section, we briefly recall the properties of dyadic decompositions in the Fourier space and give some elements of the Littlewood-Paley theory. The complete details can be found in [4], [5] and [10]. Let $\mathcal{F}$ and $\mathcal{F}^{-1}$ be the Fourier transform and its inverse, and that we also write $\widehat{u}=\mathcal{F} u$. For any $d \in \mathbb{N}^{*}$ and $0<r<R$, we denote

$$
B_{d}(0, R)=\left\{\xi \in \mathbb{R}^{d}|| \xi \mid \leq R\right\} \quad \text { and } \quad C_{d}(r, R)=\left\{\xi \in \mathbb{R}^{d}|r \leq| \xi \mid \leq R\right\} .
$$

The following Bernstein lemma gives important properties of a distribution $u$ when its Fourier transform is well localized. We refer the reader to [5] for the proof of this lemma. 
Lemma 2.1. Let $k \in \mathbb{N}, d \in \mathbb{N}^{*}$ and $r_{1}, r_{2} \in \mathbb{R}$ satisfy $0<r_{1}<r_{2}$. There exists a constant $C>0$ such that, for any $a, b \in \mathbb{R}, 1 \leq a \leq b \leq+\infty$, for any $\lambda>0$ and for any $u \in L^{a}\left(\mathbb{R}^{d}\right)$, we have

$$
\operatorname{supp}(\widehat{u}) \subset B_{d}\left(0, r_{1} \lambda\right) \Longrightarrow \sup _{|\alpha|=k}\left\|\partial^{\alpha} u\right\|_{L^{b}} \leq C^{k} \lambda^{k+d\left(\frac{1}{a}-\frac{1}{b}\right)}\|u\|_{L^{a}},
$$

and

$$
\operatorname{supp}(\widehat{u}) \subset C_{d}\left(r_{1} \lambda, r_{2} \lambda\right) \quad \Longrightarrow \quad C^{-k} \lambda^{k}\|u\|_{L^{a}} \leq \sup _{|\alpha|=k}\left\|\partial^{\alpha} u\right\|_{L^{a}} \leq C^{k} \lambda^{k}\|u\|_{L^{a}}
$$

Let $\psi$ be an even smooth function in $C_{0}^{\infty}(\mathbb{R})$, whose support is contained in the ball $B_{1}\left(0, \frac{4}{3}\right)$, such that $\psi$ is equal to 1 on a neighborhood of the ball $B_{1}\left(0, \frac{3}{4}\right)$. Let

$$
\varphi(z)=\psi\left(\frac{z}{2}\right)-\psi(z) \text {. }
$$

Then, the support of $\varphi$ is contained in the ring $C_{1}\left(\frac{3}{4}, \frac{8}{3}\right)$, and $\varphi$ is identically equal to 1 on the ring $C_{1}\left(\frac{4}{3}, \frac{3}{2}\right)$. The functions $\psi$ and $\varphi$ allow us to define a dyadic partition of $\mathbb{R}^{d}, d \in \mathbb{N}^{*}$, as follows

$$
\forall z \in \mathbb{R}, \quad \psi(z)+\sum_{j \in \mathbb{N}} \varphi\left(2^{-j} z\right)=1 .
$$

Moreover, this decomposition is almost orthogonal, in the sense that, if $\left|j-j^{\prime}\right| \geq 2$, then

$$
\operatorname{supp} \varphi\left(2^{-j}(\cdot)\right) \cap \operatorname{supp} \varphi\left(2^{-j^{\prime}}(\cdot)\right)=\emptyset \text {. }
$$

We introduce the following dyadic frequency cut-off operators. We refer to [4] and [5] for more details.

Definition 2.2. For any $d \in \mathbb{N}^{*}$ and for any tempered distribution $u \in \mathcal{S}^{\prime}\left(\mathbb{R}^{d}\right)$, we set

$$
\begin{aligned}
& \Delta_{q} u=\mathcal{F}^{-1}\left(\varphi\left(2^{-q}|\xi|\right) \widehat{u}(\xi)\right), \quad \forall q \in \mathbb{N}, \\
& \Delta_{-1} u=\mathcal{F}^{-1}(\psi(|\xi|) \widehat{u}(\xi)) \text {, } \\
& \Delta_{q} u=0 \\
& S_{q} u=\sum_{q^{\prime} \leq q-1} \Delta_{q^{\prime}} u, \quad \forall q \geq 1 .
\end{aligned}
$$

Using the properties of $\psi$ and $\varphi$, one can prove that for any tempered distribution $u \in \mathcal{S}^{\prime}\left(\mathbb{R}^{d}\right)$, we have

$$
u=\sum_{q \geq-1} \Delta_{q} u \quad \text { in } \quad \mathcal{S}^{\prime}\left(\mathbb{R}^{d}\right)
$$

and the (isotropic) nonhomogeneous Sobolev spaces $H^{s}\left(\mathbb{R}^{d}\right)$, with $s \in \mathbb{R}$, can be characterized as follows

Proposition 2.3. Let $d \in \mathbb{N}^{*}, s \in \mathbb{R}$ and $u \in H^{s}\left(\mathbb{R}^{d}\right)$. Then,

$$
\|u\|_{H^{s}}:=\left(\int_{\mathbb{R}^{d}}\left(1+|\xi|^{2}\right)^{s}|\widehat{u}(\xi)|^{2} d \xi\right)^{\frac{1}{2}} \sim\left(\sum_{q \geq-1} 2^{2 q s}\left\|\Delta_{q} u\right\|_{L^{2}}^{2}\right)^{\frac{1}{2}}
$$

Moreover, there exists a square-summable sequence of positive numbers $\left\{c_{q}(u)\right\}$ with $\sum_{q} c_{q}(u)^{2}=1$, such that

$$
\left\|\Delta_{q} u\right\|_{L^{2}} \leq c_{q}(u) 2^{-q s}\|u\|_{H^{s}} .
$$

In what follows, we also use separate dyadic decompositions in the horizontal and vertical directions.

Definition 2.4. For any tempered distribution $u \in \mathcal{S}^{\prime}\left(\mathbb{R}^{3}\right)$, we set

$$
\begin{aligned}
& \Delta_{q}^{h} u=\mathcal{F}^{-1}\left(\varphi\left(2^{-q}\left|\xi_{h}\right|\right) \widehat{u}(\xi)\right), \quad \Delta_{q}^{v} u=\mathcal{F}^{-1}\left(\varphi\left(2^{-q}\left|\xi_{3}\right|\right) \widehat{u}(\xi)\right), \quad \forall q \in \mathbb{N}, \\
& \Delta_{-1}^{h} u=\mathcal{F}^{-1}\left(\psi\left(\left|\xi_{h}\right|\right) \widehat{u}(\xi)\right), \quad \Delta_{-1}^{v} u=\mathcal{F}^{-1}\left(\psi\left(\left|\xi_{3}\right|\right) \widehat{u}(\xi)\right), \\
& \Delta_{q}^{h} u=0, \quad \quad \Delta_{q}^{v} u=0, \quad \forall q \leq-2, \\
& S_{q}^{h} u=\sum_{q^{\prime} \leq q-1} \Delta_{q^{\prime}}^{h} u, \quad S_{q}^{v} u=\sum_{q^{\prime} \leq q-1} \Delta_{q^{\prime}}^{v} u, \quad \forall q \geq 1 .
\end{aligned}
$$




\section{Exponential DECAY FOR THE 2D Limiting SyStem}

In this section, we prove the exponential decay in time of the solution of the 2D limiting system (1.3). We recall that throughout this paper, for any vector field $\bar{u}=\left(\bar{u}^{1}, \bar{u}^{2}, \bar{u}^{3}\right)$ independent of the vertical variable $x_{3}$, we denote by $\bar{w}$ the associated horizontal vorticity, $\bar{w}=\partial_{1} \bar{u}^{2}-\partial_{2} \bar{u}^{1}$. We also set $\gamma=\sqrt{2 \beta}$ to simplify the notation.

Lemma 3.1. Let $\bar{u}_{0}=\left(\bar{u}_{0}^{1}\left(x_{1}, x_{2}\right), \bar{u}_{0}^{2}\left(x_{1}, x_{2}\right), \bar{u}_{0}^{3}\left(x_{1}, x_{2}\right)\right) \in \mathbf{L}^{2}\left(\mathbb{R}_{h}^{2}\right)$ be a divergence-free vector field, the horizontal vorticity of which

$$
\bar{w}_{0}=\partial_{1} \bar{u}_{0}^{2}-\partial_{2} \bar{u}_{0}^{1} \in L^{2}\left(\mathbb{R}_{h}^{2}\right) \cap L^{\infty}\left(\mathbb{R}_{h}^{2}\right) .
$$

Then, the system (1.3), with initial data $\bar{u}_{0}$, has a unique, global solution

$$
\bar{u} \in \mathbf{C}\left(\mathbb{R}_{+}, \mathbf{L}^{2}\left(\mathbb{R}_{h}^{2}\right)\right) \cap \mathbf{L}^{\infty}\left(\mathbb{R}_{+}, \mathbf{L}^{2}\left(\mathbb{R}_{h}^{2}\right)\right) .
$$

Moreover,

(i) There exists a constant $C>0$ such that, for any $p \geq 2$ and for any $t>0$, we have

$$
\begin{gathered}
\left\|\nabla_{h} \bar{u}^{h}(t)\right\|_{\mathbf{L}^{p}\left(\mathbb{R}_{h}^{2}\right)} \leq C M p e^{-\gamma t} \\
\left\|\bar{u}^{h}(t)\right\|_{\mathbf{L}^{p}\left(\mathbb{R}_{h}^{2}\right)} \leq C M e^{-\gamma t}
\end{gathered}
$$

where

$$
M=\max \left\{\left\|\bar{u}_{0}^{h}\right\|_{\mathbf{L}^{2}\left(\mathbb{R}_{h}^{2}\right)},\left\|\bar{w}_{0}\right\|_{L^{2}\left(\mathbb{R}_{h}^{2}\right)},\left\|\bar{w}_{0}\right\|_{L^{\infty}\left(\mathbb{R}_{h}^{2}\right)}\right\} .
$$

(ii) For any $p \geq 2$, if $\bar{u}_{0}^{3} \in \mathbf{L}^{p}\left(\mathbb{R}_{h}^{2}\right)$, then,

$$
\left\|\bar{u}^{3}(t)\right\|_{L^{p}\left(\mathbb{R}_{h}^{2}\right)} \leq\left\|\bar{u}_{0}^{3}\right\|_{L^{p}\left(\mathbb{R}_{h}^{2}\right)} e^{-\gamma t} .
$$

\section{Proof}

In (1.3), the first two components of $\bar{u}$ satisfy a two-dimensional damped Euler system. Then, thanks to the Yudovitch theorem [28] (see also [5]), this system has a unique solution

$$
\bar{u}^{h} \in \mathbf{C}\left(\mathbb{R}_{+}, \mathbf{L}^{2}\left(\mathbb{R}_{h}^{2}\right)\right) \cap \mathbf{L}^{\infty}\left(\mathbb{R}_{+}, \mathbf{L}^{2}\left(\mathbb{R}_{h}^{2}\right)\right)
$$

such that the horizontal vorticity $\bar{w} \in \mathbf{L}^{\infty}\left(\mathbb{R}_{+}, \mathbf{L}^{2}\left(\mathbb{R}_{h}^{2}\right)\right) \cap \mathbf{L}^{\infty}\left(\mathbb{R}_{+}, \mathbf{L}^{\infty}\left(\mathbb{R}_{h}^{2}\right)\right)$. Since the third component $\bar{u}^{3}$ satisfies a linear transport-type equation, we can deduce the existence and uniqueness of the solution $\bar{u}$ of the limiting system (1.3).

By definition, the horizontal vorticity $\bar{w}$ satisfies the following equation

$$
\partial_{t} \bar{w}+\gamma \bar{w}+\bar{u}^{h} \cdot \nabla_{h} \bar{w}=0
$$

Taking the $L^{2}$ scalar product of (3.4) with $|\bar{w}|^{p-2} \bar{w}$, we get

$$
\frac{1}{p} \frac{d}{d t}\|\bar{w}\|_{L^{p}\left(\mathbb{R}_{h}^{2}\right)}^{p}+\gamma\|\bar{w}\|_{L^{p}\left(\mathbb{R}_{h}^{2}\right)}^{p}=0 .
$$

By using an interpolation between $L^{2}\left(\mathbb{R}_{h}^{2}\right)$ and $L^{\infty}\left(\mathbb{R}_{h}^{2}\right)$, we deduce from the above equation that

$$
\|\bar{w}(t)\|_{L^{p}\left(\mathbb{R}_{h}^{2}\right)} \leq\left\|\bar{w}_{0}\right\|_{L^{p}\left(\mathbb{R}_{h}^{2}\right)} \mathrm{e}^{-\gamma t} \leq C\left\|\bar{w}_{0}\right\|_{L^{2}\left(\mathbb{R}_{h}^{2}\right)}^{\frac{2}{p}}\left\|\bar{w}_{0}\right\|_{L^{\infty}\left(\mathbb{R}_{h}^{2}\right)}^{1-\frac{2}{p}} \mathrm{e}^{-\gamma t} \leq C M \mathrm{e}^{-\gamma t} .
$$

We recall that $\nabla_{h} \bar{u}^{h}=R \bar{w}$ where $R$ is an homogeneous Caldéron-Zygmund operator of order 0. According to [[5], Theorem 3.1.1], for any $p>1$ and for any $t>0$,

$$
\left\|\nabla_{h} \bar{u}^{h}(t)\right\|_{L^{p}\left(\mathbb{R}_{h}^{2}\right)} \leq C \frac{p^{2}}{p-1}\|\bar{w}(t)\|_{L^{p}\left(\mathbb{R}_{h}^{2}\right)},
$$

which implies (3.1), for any $p \geq 2$. In particular, we have

$$
\left\|\bar{u}^{h}(t)\right\|_{\dot{\mathbf{H}}^{1}\left(\mathbb{R}_{h}^{2}\right)}=\left\|\nabla_{h} \bar{u}^{h}(t)\right\|_{\mathbf{L}^{2}\left(\mathbb{R}_{h}^{2}\right)}=\|\bar{w}(t)\|_{L^{2}\left(\mathbb{R}_{h}^{2}\right)} \leq C M \mathrm{e}^{-\gamma t} .
$$

We remark that Inequality (3.2), in the case where $p=2$, directly comes from the energy estimate for the damped Euler systems. So, using the Sobolev embedding $\dot{\mathbf{H}}^{\frac{p-2}{p}}\left(\mathbb{R}_{h}^{2}\right) \hookrightarrow \mathbf{L}^{p}\left(\mathbb{R}_{h}^{2}\right)$ and the fact that $\dot{\mathbf{H}}^{\frac{p-2}{p}}\left(\mathbb{R}_{h}^{2}\right)$ is an interpolated space between $\mathbf{L}^{2}\left(\mathbb{R}_{h}^{2}\right)$ and $\dot{\mathbf{H}}^{1}\left(\mathbb{R}_{h}^{2}\right)$, we deduce (3.2) from Estimate (3.6), when $p \geq 2$. Since the vertical component $\bar{u}^{3}$ satisfies the same linear transport equation as $\bar{w}$, Inequality (3.3) can be proved in the same way as (3.5). 
Lemma 3.2. Let $r>1$. There is a constant $C>0$ such that, for any divergence-free vector fields $\bar{u}$ in $\mathbf{L}^{2}\left(\mathbb{R}_{h}^{2}\right)$, the horizontal gradient $\nabla_{h} \bar{u}^{h} \in \mathbf{L}^{\infty}\left(\mathbb{R}_{h}^{2}\right)$ and the vorticity $\bar{w}=\partial_{1} \bar{u}^{2}-\partial_{2} \bar{u}^{1} \in H^{r}\left(\mathbb{R}_{h}^{2}\right)$, we have

$$
\left|\left\langle\Delta_{q}^{h}\left(\bar{u}^{h} \cdot \nabla_{h} \bar{w}\right) \mid \Delta_{q}^{h} \bar{w}\right\rangle\right| \leq C d_{q} 2^{-2 q r}\left(\left\|\nabla_{h} \bar{u}^{h}\right\|_{\mathbf{L}^{\infty}}+\|\bar{w}\|_{L^{\infty}}\right)\|\bar{w}\|_{H^{r}}^{2} .
$$

where $\left(d_{q}\right)_{q \geq-1}$ is a summable sequence of positive numbers, the $\ell^{1}$-norm of which is independent of $\bar{u}$.

In order to prove Lemma 3.2, we need the following estimate (the proof of which can be found in [24]). Let $[.,$.$] denote the usual commutator.$

Lemma 3.3. Let $d \in \mathbb{N}^{*}$. There exists a constant $C>0$ such that, for any tempered distributions $u, v$ in $\mathcal{S}^{\prime}\left(\mathbb{R}^{d}\right)$, we have

$$
\left\|\left[\Delta_{q}, u\right] v\right\|_{L^{2}}:=\left\|\Delta_{q}(u v)-u \Delta_{q} v\right\|_{L^{2}} \leq C 2^{-q}\|\nabla u\|_{L^{\infty}}\|v\|_{L^{2}} .
$$

\section{Proof of Lemma 3.2}

First of all, using the anisotropic Bony decomposition into paraproducts and remainders as in Definition 2.4, we can write

$$
\left|\left\langle\Delta_{q}^{h}\left(\bar{u}^{h} \cdot \nabla_{h} \bar{w}\right) \mid \Delta_{q}^{h} \bar{w}\right\rangle\right| \leq I_{q}^{1}(\bar{w})+I_{q}^{2}(\bar{w})
$$

where

and

$$
I_{q}^{1}(\bar{w})=\left|\left\langle\Delta_{q}^{h}\left(\sum_{q^{\prime}-q \geq N} S_{q^{\prime}+2}^{h}\left(\nabla_{h} \bar{w}\right) \Delta_{q^{\prime}}^{h} \bar{u}^{h}\right) \mid \Delta_{q}^{h} \bar{w}\right\rangle\right|,
$$

$$
I_{q}^{2}(\bar{w})=\mid\left\langle\Delta_{q}^{h}\left(\sum_{\left|q^{\prime}-q\right| \leq N} S_{q^{\prime}-1}^{h} \bar{u}^{h} \Delta_{q^{\prime}}^{h}\left(\nabla_{h} \bar{w}\right)\right) \mid \Delta_{q}^{h} \bar{w}\right\rangle,
$$

and where $N>0$ is a fixed large enough integer.

Using Cauchy-Schwarz and Hölder inequalities, we get

$$
I_{q}^{1}(\bar{w}) \leq \sum_{q^{\prime}-q \geq N}\left\|S_{q^{\prime}+2}^{h}\left(\nabla_{h} \bar{w}\right)\right\|_{\mathbf{L}^{\infty}}\left\|\Delta_{q^{\prime}}^{h} \bar{u}^{h}\right\|_{\mathbf{L}^{2}}\left\|\Delta_{q}^{h} \bar{w}\right\|_{L^{2}} .
$$

The Bernstein lemma 2.1 implies that

and

$$
\left\|S_{q^{\prime}+2}^{h}\left(\nabla_{h} \bar{w}\right)\right\|_{\mathbf{L}^{\infty}} \leq C 2^{q^{\prime}}\left\|S_{q^{\prime}+2}^{h} \bar{w}\right\|_{L^{\infty}} \leq C 2^{q^{\prime}}\|\bar{w}\|_{L^{\infty}},
$$

$$
\left\|\Delta_{q^{\prime}}^{h} \bar{u}^{h}\right\|_{\mathbf{L}^{2}} \leq C 2^{-q^{\prime}}\left\|\Delta_{q^{\prime}}^{h} \nabla_{h} \bar{u}^{h}\right\|_{\mathbf{L}^{2}} .
$$

Taking into account the fact that $\bar{w}=\partial_{1} \bar{u}^{2}-\partial_{2} \bar{u}^{1}$ and $\operatorname{div}_{h} \bar{u}^{h}=0$, using an integration by parts, we easily obtain

$$
\begin{aligned}
\left\|\Delta_{q^{\prime}}^{h} \bar{w}\right\|_{L^{2}}^{2} & =\left\|\Delta_{q^{\prime}}^{h} \partial_{1} \bar{u}^{2}\right\|_{L^{2}}^{2}+\left\|\Delta_{q^{\prime}}^{h} \partial_{2} \bar{u}^{1}\right\|_{L^{2}}^{2}-2\left\langle\Delta_{q^{\prime}}^{h} \partial_{1} \bar{u}^{2} \mid \Delta_{q^{\prime}}^{h} \partial_{2} \bar{u}^{1}\right\rangle \\
& =\left\|\Delta_{q^{\prime}}^{h} \partial_{1} \bar{u}^{2}\right\|_{L^{2}}^{2}+\left\|\Delta_{q^{\prime}}^{h} \partial_{2} \bar{u}^{1}\right\|_{L^{2}}^{2}+\left\|\Delta_{q^{\prime}}^{h} \partial_{1} \bar{u}^{1}\right\|_{L^{2}}^{2}+\left\|\Delta_{q^{\prime}}^{h} \partial_{2} \bar{u}^{2}\right\|_{L^{2}}^{2} \\
& =\left\|\Delta_{q^{\prime}}^{h} \nabla_{h} \bar{u}^{h}\right\|_{L^{2}}^{2} .
\end{aligned}
$$

Therefore, using Proposition 2.3, we deduce from (3.9) that

$$
\begin{aligned}
I_{q}^{1}(\bar{w}) & \leq C \sum_{q^{\prime}-q \geq N}\|\bar{w}\|_{L^{\infty}}\left\|\Delta_{q^{\prime}}^{h} \bar{w}\right\|_{L^{2}}\left\|\Delta_{q}^{h} \bar{w}\right\|_{L^{2}} \\
& \leq C\left(c_{q}(\bar{w}) \sum_{q^{\prime}-q \geq N} c_{q^{\prime}}(\bar{w}) 2^{-\left(q^{\prime}-q\right) r}\right) 2^{-2 q r}\|\bar{w}\|_{L^{\infty}}\|\bar{w}\|_{H^{r}}^{2},
\end{aligned}
$$

where $\left(c_{q}(\bar{w})\right)_{q \geq-1}$ is a square-summable sequence of positive numbers such that $\sum_{q} c_{q}(\bar{w})^{2}=1$. We remark that $\left(2^{-q r}\right)_{q \geq-N}$ is a summable sequence. So, as a convolution product of a $\ell^{2}$-sequence and a $\ell^{1}$-sequence,

is square-summable. Let

$$
\left(\sum_{q^{\prime}-q \geq N} c_{q^{\prime}}(\bar{w}) 2^{-\left(q^{\prime}-q\right) r}\right)_{q}
$$

$$
d_{q}=c_{q}(\bar{w}) \sum_{q^{\prime}-q \geq N} c_{q^{\prime}}(\bar{w}) 2^{-\left(q^{\prime}-q\right) r}
$$


It is clear that $\left(d_{q}\right)_{q \geq-1}$ is a summable sequence of positive numbers. Thus, we have

$$
I_{q}^{1}(\bar{w}) \leq C d_{q} 2^{-2 q r}\|\bar{w}\|_{L^{\infty}}\|\bar{w}\|_{H^{r}}^{2} .
$$

In order to estimate $I_{q}^{2}(\bar{w})$, we write

$$
I_{q}^{2}(\bar{w}) \leq I_{q}^{2 A}(\bar{w})+I_{q}^{2 B}(\bar{w})+I_{q}^{2 C}(\bar{w}),
$$

where

and

$$
\begin{gathered}
I_{q}^{2 A}(\bar{w})=\left|\left\langle S_{q}^{h} \bar{u}^{h} \cdot \nabla_{h} \Delta_{q}^{h} \bar{w} \mid \Delta_{q}^{h} \bar{w}\right\rangle\right|, \\
I_{q}^{2 B}(\bar{w})=\left|\left\langle\sum_{\left|q^{\prime}-q\right| \leq N}\left(S_{q}^{h}-S_{q^{\prime}-1}^{h}\right) \bar{u}^{h} \cdot \nabla_{h} \Delta_{q^{\prime}}^{h} \bar{w} \mid \Delta_{q}^{h} \bar{w}\right\rangle\right|
\end{gathered}
$$

$$
I_{q}^{2 C}(\bar{w})=\left|\left\langle\sum_{\left|q^{\prime}-q\right| \leq N}\left[\Delta_{q}^{h}, S_{q^{\prime}-1}^{h} \bar{u}^{h}\right] \nabla_{h} \Delta_{q^{\prime}}^{h} \bar{w} \mid \Delta_{q}^{h} \bar{w}\right\rangle\right|
$$

Using integrations by parts, we get

$$
I_{q}^{2 A}(\bar{w})=\frac{1}{2}\left|\left\langle S_{q}^{h}\left(\operatorname{div}_{h} \bar{u}^{h}\right) \Delta_{q}^{h} \bar{w} \mid \Delta_{q}^{h} \bar{w}\right\rangle\right|=0 .
$$

We remark that when $q, q^{\prime} \geq 1, S_{q}^{h}-S_{q^{\prime}-1}^{h}$ does not contain the low Fourier frequencies. So, $I_{q}^{2 B}(\bar{w})$ can be bounded in the same way as $I_{q}^{1}(\bar{w})$, and we have

$$
I_{q}^{2 B}(\bar{w}) \leq C d_{q} 2^{-2 q r}\|\bar{w}\|_{L^{\infty}}\|\bar{w}\|_{H^{r}}^{2} .
$$

Finally, using Lemma 3.3 and the same calculations as for $I_{q}^{1}(\bar{w})$ (since $r>1$ ), we obtain

$$
\begin{aligned}
& I_{q}^{2 C}(\bar{w}) \leq C 2^{-q} \sum_{\left|q^{\prime}-q\right| \leq N}\left\|S_{q^{\prime}-1}^{h} \nabla_{h} \bar{u}^{h}\right\|_{\mathbf{L}^{\infty}}\left\|\nabla_{h} \Delta_{q^{\prime}}^{h} \bar{w}\right\|_{\mathbf{L}^{2}}\left\|\Delta_{q}^{h} \bar{w}\right\|_{L^{2}} \\
& \leq C 2^{-2 q r}\left\|\nabla_{h} \bar{u}^{h}\right\|_{\mathbf{L}^{\infty}}\|\bar{w}\|_{H^{r}}^{2}\left(c_{q}(\bar{w}) \sum_{\left|q^{\prime}-q\right| \leq N} c_{q^{\prime}}(\bar{w}) 2^{-\left(q^{\prime}-q\right)(r-1)}\right) \\
& \leq C d_{q} 2^{-2 q r}\left\|\nabla_{h} \bar{u}^{h}\right\|_{\mathbf{L}^{\infty}}\|\bar{w}\|_{H^{r}}^{2} .
\end{aligned}
$$

From (3.10)-(3.14), we easily deduce (3.7) and Lemma 3.2 is proved.

Lemma 3.4. Let $r>1$. There exists a constant $C_{r}>0$ such that, for any divergence-free vector fields $\bar{u}$ in $\mathbf{L}^{2}\left(\mathbb{R}_{h}^{2}\right)$, the horizontal gradient $\nabla_{h} \bar{u}^{h} \in \mathbf{L}^{\infty}\left(\mathbb{R}_{h}^{2}\right)$ and the vorticity $\bar{w}=\partial_{1} \bar{u}^{2}-\partial_{2} \bar{u}^{1} \in H^{r}\left(\mathbb{R}_{h}^{2}\right)$, we have

$$
\left\|\nabla_{h} \bar{u}^{h}\right\|_{\mathbf{L}^{\infty}\left(\mathbb{R}_{h}^{2}\right)} \leq C_{r}\|\bar{w}\|_{L^{\infty}\left(\mathbb{R}_{h}^{2}\right)} \ln \left(e+\frac{\|\bar{w}\|_{H^{r}\left(\mathbb{R}_{h}^{2}\right)}}{\|\bar{w}\|_{L^{\infty}\left(\mathbb{R}_{h}^{2}\right)}}\right) .
$$

The lemma 3.4 is only a special case of [[5], Theorem 3.3.2]. We refer to [5] for a proof of this lemma.

Lemma 3.5. Let $r>1$. Under the hypotheses of Lemma 3.1 and the additional hypothesis that $\bar{w}_{0}$ belongs to $H^{r}\left(\mathbb{R}_{h}^{2}\right)$, there exist positive constants $C_{1}$ and $C_{2}$ depending on $\gamma$ and $\left\|\bar{w}_{0}\right\|_{H^{r}\left(\mathbb{R}^{2}\right)}$ such that

and

$$
\|\bar{w}(t)\|_{H^{r}\left(\mathbb{R}_{h}^{2}\right)} \leq C_{1} e^{-\gamma t},
$$

\section{Proof}

In what follows, we use $C$ to refer to a generic positive constant that may change from line to line. For any $r>1$, using Lemma 3.2, we get the following energy estimate in the Sobolev $H^{r}$-norm:

$$
\frac{1}{2} \frac{d}{d t}\|\bar{w}(t)\|_{H^{r}}^{2}+\gamma\|\bar{w}(t)\|_{H^{r}}^{2} \leq C\left(\|\bar{w}(t)\|_{L^{\infty}}+\left\|\nabla_{h} \bar{u}^{h}(t)\right\|_{\mathbf{L}^{\infty}}\right)\|\bar{w}(t)\|_{H^{r}}^{2} .
$$

Taking into account Estimate (3.15), we rewrite (3.18) as follows

$$
\frac{d}{d t}\|\bar{w}(t)\|_{H^{r}}+\gamma\|\bar{w}(t)\|_{H^{r}} \leq C\|\bar{w}\|_{L^{\infty}}\left(1+\ln \left(e+\frac{\|\bar{w}\|_{H^{r}}}{\|\bar{w}\|_{L^{\infty}}}\right)\right)\|\bar{w}(t)\|_{H^{r}} .
$$


From (3.5), we have $\|\bar{w}(t)\|_{L^{p}} \leq C M \mathrm{e}^{-\gamma t}$, where

$$
M=\max \left\{\left\|\bar{u}_{0}^{h}\right\|_{\mathbf{L}^{2}\left(\mathbb{R}_{h}^{2}\right)},\left\|\bar{w}_{0}\right\|_{L^{2}\left(\mathbb{R}_{h}^{2}\right)},\left\|\bar{w}_{0}\right\|_{L^{\infty}\left(\mathbb{R}_{h}^{2}\right)}\right\} .
$$

Since $C$ and $M$ do not depend on $p$, we have

$$
\|\bar{w}(t)\|_{L^{\infty}} \leq C M \mathrm{e}^{-\gamma t}
$$

We remark that $x \ln \left(e+\frac{\alpha}{x}\right), \alpha>0$, is an increasing function. We deduce from (3.19) that

$$
\frac{d}{d t}\|\bar{w}(t)\|_{H^{r}}+\gamma\|\bar{w}(t)\|_{H^{r}} \leq C M \mathrm{e}^{-\gamma t}\left(1+\ln \left(e+\frac{\|\bar{w}\|_{H^{r}} \mathrm{e}^{\gamma t}}{C M}\right)\right)\|\bar{w}(t)\|_{H^{r}} .
$$

Therefore, considering $y(t)=(C M)^{-1}\|\bar{w}(t)\|_{H^{r}} e^{\gamma t}$, we get from (3.20) that

$$
y^{\prime}(t) \leq C M e^{-\gamma t} y(t)(1+\ln (e+y(t))),
$$

which implies

$$
\frac{y^{\prime}(t)}{(e+y(t))(1+\ln (e+y(t)))} \leq C M e^{-\gamma t} .
$$

Integrating (3.21) with respect to $t$, we obtain

$$
\ln (1+\ln (e+y(t))) \leq \ln (1+\ln (e+y(0)))+\frac{C M}{\gamma} .
$$

Choosing

$$
C_{1}=C M\left(e+\left\|\bar{w}_{0}\right\|_{H^{r}}\right)^{2 e^{\frac{C M}{\gamma}}},
$$

we get

$$
\|\bar{w}(t)\|_{H^{r}} \leq C_{1} e^{-\gamma t} .
$$

Combining the above estimate with (3.15) and using again the fact that $x \ln \left(e+\frac{\alpha}{x}\right)$ is an increasing function, we obtain the existence of a positive constant $C_{2}$, depending on $\gamma$ and $\left\|\bar{w}_{0}\right\|_{H^{r}}$, such that

$$
\left\|\nabla_{h} \bar{u}^{h}(t)\right\|_{\mathbf{L}^{\infty}} \leq C_{2} e^{-\gamma t} .
$$

In order to prove Theorem 1.2, we finally need the following lemma.

Lemma 3.6. Let $\sigma>2$. For any divergence-free vector fields $\bar{u}=\left(\bar{u}^{1}\left(x_{1}, x_{2}\right), \bar{u}^{2}\left(x_{1}, x_{2}\right), \bar{u}^{3}\left(x_{1}, x_{2}\right)\right)$ in $\mathbf{H}^{\sigma}\left(\mathbb{R}_{h}^{2}\right)$, there exist a constant $C>0$ independent of $\bar{u}$, a summable sequence $\left(d_{q}\left(\bar{u}^{h}\right)\right)_{q \geq-1}$ of positive numbers such that

$$
\left|\left\langle\Delta_{q}^{h}\left(\bar{u}^{h} \cdot \nabla_{h} \bar{u}^{h}\right) \mid \Delta_{q}^{h} \bar{u}^{h}\right\rangle\right| \leq C d_{q}\left(\bar{u}^{h}\right) 2^{-2 q \sigma}\left\|\nabla_{h} \bar{u}^{h}\right\|_{\mathbf{L}^{\infty}}\left\|\bar{u}^{h}\right\|_{\mathbf{H}^{\sigma}}^{2},
$$

and a summable sequence $\left(d_{q}(\bar{u})\right)_{q \geq-1}$ of positive numbers such that

$$
\left|\left\langle\Delta_{q}^{h}\left(\bar{u}^{h} \cdot \nabla_{h} \bar{u}^{3}\right) \mid \Delta_{q}^{h} \bar{u}^{3}\right\rangle\right| \leq C d_{q}(\bar{u}) 2^{-2 q \sigma}\left\|\bar{u}^{h}\right\|_{\mathbf{H}^{\sigma}}\left\|\bar{u}^{3}\right\|_{H^{\sigma}}^{2} .
$$

\section{Proof}

We use the similar Bony decomposition as in the proof of Lemma 3.2, with $\bar{w}$ replaced by $\bar{u}^{h}$.

$$
\left|\left\langle\Delta_{q}^{h}\left(\bar{u}^{h} \cdot \nabla_{h} \bar{u}^{h}\right) \mid \Delta_{q}^{h} \bar{u}^{h}\right\rangle\right| \leq I_{q}^{1}\left(\bar{u}^{h}\right)+I_{q}^{2 A}\left(\bar{u}^{h}\right)+I_{q}^{2 B}\left(\bar{u}^{h}\right)+I_{q}^{2 C}\left(\bar{u}^{h}\right) .
$$

Then, using Young's inequality, Bernstein lemma 2.1 and Proposition 2.3, we have

$$
\begin{aligned}
I_{q}^{1}\left(\bar{u}^{h}\right) & =\left|\left\langle\Delta_{q}^{h}\left(\sum_{q^{\prime}-q \geq N} S_{q^{\prime}+2}^{h}\left(\nabla_{h} \bar{u}^{h}\right) \Delta_{q^{\prime}}^{h} \bar{u}^{h}\right) \mid \Delta_{q}^{h} \bar{u}^{h}\right\rangle\right| \\
& \leq \sum_{q^{\prime}-q \geq N}\left\|S_{q^{\prime}+2}^{h}\left(\nabla_{h} \bar{u}^{h}\right)\right\|_{\mathbf{L}^{\infty}}\left\|\Delta_{q^{\prime}}^{h} \bar{u}^{h}\right\|_{\mathbf{L}^{2}}\left\|\Delta_{q}^{h} \bar{u}^{h}\right\|_{L^{2}} \\
& \leq C \sum_{q^{\prime}-q \geq N}\left\|\nabla_{h} \bar{u}^{h}\right\|_{\mathbf{L}^{\infty}}\left(c_{q^{\prime}}\left(\bar{u}^{h}\right) 2^{-q^{\prime} \sigma}\left\|\bar{u}^{h}\right\|_{\mathbf{H}^{\sigma}}\right)\left(c_{q}\left(\bar{u}^{h}\right) 2^{-q \sigma}\left\|\bar{u}^{h}\right\|_{\mathbf{H}^{\sigma}}\right) \\
& =C\left(c_{q}\left(\bar{u}^{h}\right) \sum_{q^{\prime}-q \geq N} c_{q^{\prime}}\left(\bar{u}^{h}\right) 2^{-\left(q^{\prime}-q\right) \sigma}\right) 2^{-2 q \sigma}\left\|\nabla_{h} \bar{u}^{h}\right\|_{\mathbf{L}^{\infty}}\left\|\bar{u}^{h}\right\|_{\mathbf{H}^{\sigma}}^{2} \\
& =C d_{q}\left(\bar{u}^{h}\right) 2^{-2 q \sigma}\left\|\nabla_{h} \bar{u}^{h}\right\|_{\mathbf{L}^{\infty}}\left\|\bar{u}^{h}\right\|_{\mathbf{H}^{\sigma}}^{2} .
\end{aligned}
$$


As in the proof of Lemma 3.2, integrations by parts imply

$$
I_{q}^{2 A}\left(\bar{u}^{h}\right)=\left|\left\langle S_{q}^{h} \bar{u}^{h} \cdot \nabla_{h} \Delta_{q}^{h} \bar{u}^{h} \mid \Delta_{q}^{h} \bar{u}^{h}\right\rangle\right|=0 .
$$

Since for any $q, q^{\prime} \geq 1, S_{q}^{h}-S_{q^{\prime}-1}^{h}$ does not contain the low Fourier frequencies, $I_{q}^{2 B}$ can be bounded in the same way as $I_{q}^{1}$, and we have

$$
I_{q}^{2 B}\left(\bar{u}^{h}\right)=\left|\left\langle\sum_{\left|q^{\prime}-q\right| \leq N}\left(S_{q}^{h}-S_{q^{\prime}-1}^{h}\right) \bar{u}^{h} \cdot \nabla_{h} \Delta_{q^{\prime}}^{h} \bar{u}^{h} \mid \Delta_{q}^{h} \bar{u}^{h}\right\rangle\right| \leq C d_{q}\left(\bar{u}^{h}\right) 2^{-2 q \sigma}\left\|\nabla_{h} \bar{u}^{h}\right\|_{\mathbf{L}^{\infty}}\left\|\bar{u}^{h}\right\|_{\mathbf{H}^{\sigma}}^{2} .
$$

The last term on the right hand side of (3.24) can be bounded exactly in the same way as in (3.14)

$$
\begin{aligned}
I_{q}^{2 C}\left(\bar{u}^{h}\right) & =\left|\left\langle\sum_{\left|q^{\prime}-q\right| \leq N}\left[\Delta_{q}^{h}, S_{q^{\prime}-1}^{h} \bar{u}^{h}\right] \nabla_{h} \Delta_{q^{\prime}}^{h} \bar{u}^{h} \mid \Delta_{q}^{h} \bar{u}^{h}\right\rangle\right| \\
& \leq C 2^{-q} \sum_{\left|q^{\prime}-q\right| \leq N}\left\|S_{q^{\prime}-1}^{h} \nabla_{h} \bar{u}^{h}\right\|_{\mathbf{L}^{\infty}}\left\|\nabla_{h} \Delta_{q^{\prime}}^{h} \bar{u}^{h}\right\|_{\mathbf{L}^{2}}\left\|\Delta_{q}^{h} \bar{u}^{h}\right\|_{L^{2}} \\
& \leq C 2^{-2 q \sigma}\left\|\nabla_{h} \bar{u}^{h}\right\|_{\mathbf{L}^{\infty}}\left\|\bar{u}^{h}\right\|_{\mathbf{H}^{\sigma}}^{2}\left(c_{q}\left(\bar{u}^{h}\right) \sum_{\left|q^{\prime}-q\right| \leq N} c_{q^{\prime}}\left(\bar{u}^{h}\right) 2^{-\left(q^{\prime}-q\right)(\sigma-1)}\right) \\
& \leq C d_{q}\left(\bar{u}^{h}\right) 2^{-2 q \sigma}\left\|\nabla_{h} \bar{u}^{h}\right\|_{\mathbf{L}^{\infty}}\left\|\bar{u}^{h}\right\|_{\mathbf{H}^{\sigma}}^{2} .
\end{aligned}
$$

Combining Estimates (3.24) to (3.28), we obtain (3.22).

Now, to prove (3.23), as in (3.24), with $\bar{u}^{h}$ replaced by $\bar{u}^{3}$, we also write

$$
\left|\left\langle\Delta_{q}^{h}\left(\bar{u}^{h} \cdot \nabla_{h} \bar{u}^{3}\right) \mid \Delta_{q}^{h} \bar{u}^{3}\right\rangle\right| \leq I_{q}^{1}\left(\bar{u}^{3}\right)+I_{q}^{2 A}\left(\bar{u}^{3}\right)+I_{q}^{2 B}\left(\bar{u}^{3}\right)+I_{q}^{2 C}\left(\bar{u}^{3}\right) .
$$

We will estimate $I_{q}^{2 A}\left(\bar{u}^{3}\right)$ and $I_{q}^{2 C}\left(\bar{u}^{3}\right)$ exactly in the same way as $I_{q}^{2 A}\left(\bar{u}^{h}\right)$ and $I_{q}^{2 C}\left(\bar{u}^{h}\right)$. We have

$$
I_{q}^{2 A}\left(\bar{u}^{3}\right)=\left|\left\langle S_{q}^{h} \bar{u}^{h} \cdot \nabla_{h} \Delta_{q}^{h} \bar{u}^{3} \mid \Delta_{q}^{h} \bar{u}^{3}\right\rangle\right|=0,
$$

and, using the inclusion $H^{\sigma-1}\left(\mathbb{R}_{h}^{2}\right) \hookrightarrow L^{\infty}\left(\mathbb{R}_{h}^{2}\right)$ for $\sigma>2$,

$$
\begin{aligned}
I_{q}^{2 C}\left(\bar{u}^{3}\right) & =\left|\left\langle\sum_{\left|q^{\prime}-q\right| \leq N}\left[\Delta_{q}^{h}, S_{q^{\prime}-1}^{h} \bar{u}^{h}\right] \nabla_{h} \Delta_{q^{\prime}}^{h} \bar{u}^{3} \mid \Delta_{q}^{h} \bar{u}^{3}\right\rangle\right| \\
& \leq C 2^{-q} \sum_{\left|q^{\prime}-q\right| \leq N}\left\|S_{q^{\prime}-1}^{h} \nabla_{h} \bar{u}^{h}\right\|_{\mathbf{L}^{\infty}}\left\|\nabla_{h} \Delta_{q^{\prime}}^{h} \bar{u}^{3}\right\|_{\mathbf{L}^{2}}\left\|\Delta_{q}^{h} \bar{u}^{3}\right\|_{L^{2}} \\
& \leq C 2^{-2 q \sigma}\left\|\nabla_{h} \bar{u}^{h}\right\|_{\mathbf{L}^{\infty}}\left\|\bar{u}^{3}\right\|_{\mathbf{H}^{\sigma}}^{2}\left(c_{q}\left(\bar{u}^{3}\right) \sum_{\left|q^{\prime}-q\right| \leq N} c_{q^{\prime}}\left(\bar{u}^{3}\right) 2^{-\left(q^{\prime}-q\right)(\sigma-1)}\right) \\
& \leq C d_{q}\left(\bar{u}^{3}\right) 2^{-2 q \sigma}\left\|\bar{u}^{h}\right\|_{\mathbf{H}^{\sigma}}\left\|\bar{u}^{3}\right\|_{\mathbf{H}^{\sigma}}^{2} .
\end{aligned}
$$

To estimate $I_{q}^{1}\left(\bar{u}^{3}\right)$, we write

$$
\begin{aligned}
I_{q}^{1}\left(\bar{u}^{3}\right) & =\left|\left\langle\Delta_{q}^{h}\left(\sum_{q^{\prime}-q \geq N} S_{q^{\prime}+2}^{h}\left(\nabla_{h} \bar{u}^{3}\right) \Delta_{q^{\prime}}^{h} \bar{u}^{h}\right) \mid \Delta_{q}^{h} \bar{u}^{3}\right\rangle\right| \\
& \leq \sum_{q^{\prime}-q \geq N}\left\|S_{q^{\prime}+2}^{h}\left(\nabla_{h} \bar{u}^{3}\right)\right\|_{\mathbf{L}^{\infty}}\left\|\Delta_{q^{\prime}}^{h} \bar{u}^{h}\right\|_{\mathbf{L}^{2}}\left\|\Delta_{q}^{h} \bar{u}^{3}\right\|_{L^{2}} \\
& \leq C \sum_{q^{\prime}-q \geq N}\left\|\nabla_{h} \bar{u}^{3}\right\|_{\mathbf{L}^{\infty}}\left(c_{q^{\prime}}\left(\bar{u}^{h}\right) 2^{-q^{\prime} \sigma}\left\|\bar{u}^{h}\right\|_{\mathbf{H}^{\sigma}}\right)\left(c_{q}\left(\bar{u}^{3}\right) 2^{-q \sigma}\left\|\bar{u}^{3}\right\|_{H^{\sigma}}\right) \\
& =C\left(c_{q}\left(\bar{u}^{3}\right) \sum_{q^{\prime}-q \geq N} c_{q^{\prime}}\left(\bar{u}^{h}\right) 2^{-\left(q^{\prime}-q\right) \sigma}\right. \\
& =C d_{q}(\bar{u}) 2^{-2 q \sigma}\left\|\bar{u}^{h}\right\|_{\mathbf{H}^{\sigma}}\left\|\bar{u}^{3}\right\|_{H^{\sigma}}^{2} .
\end{aligned}
$$


Finally, the term $I_{q}^{2 B}\left(\bar{u}^{3}\right)$ can be bounded in the same way as $I_{q}^{1}\left(\bar{u}^{3}\right)$,

$$
I_{q}^{2 B}\left(\bar{u}^{3}\right)=\left|\left\langle\sum_{\left|q^{\prime}-q\right| \leq N}\left(S_{q}^{h}-S_{q^{\prime}-1}^{h}\right) \bar{u}^{h} \cdot \nabla_{h} \Delta_{q^{\prime}}^{h} \bar{u}^{3} \mid \Delta_{q}^{h} \bar{u}^{3}\right\rangle\right| \leq C d_{q}(\bar{u}) 2^{-2 q \sigma}\left\|\bar{u}^{h}\right\|_{\mathbf{H}^{\sigma}}\left\|\bar{u}^{3}\right\|_{H^{\sigma}}^{2} .
$$

Combining Estimates (3.29) to (3.33), we obtain (3.23).

\section{Proof of Theorem 1.2}

We recall that $C$ to refer to a generic positive constant that may change from line to line. Since $\bar{u}^{h}$ satisfies a two-dimensional damped Euler system, using Lemma 3.6, we deduce the following energy estimate in the Sobolev $\mathbf{H}^{\sigma}$-norm for $\bar{u}^{h}$

$$
\frac{1}{2} \frac{d}{d t}\left\|\bar{u}^{h}(t)\right\|_{\mathbf{H}^{\sigma}}^{2}+\gamma\left\|\bar{u}^{h}(t)\right\|_{\mathbf{H}^{\sigma}}^{2} \leq C\left\|\nabla_{h} \bar{u}^{h}(t)\right\|_{\mathbf{L}^{\infty}}\left\|\bar{u}^{h}(t)\right\|_{\mathbf{H}^{\sigma}}^{2} .
$$

Since $\bar{u}_{0}^{h} \in \mathbf{H}^{\sigma}\left(\mathbb{R}_{h}^{2}\right), \sigma>2$, we have $\bar{w}_{0}=\partial_{1} \bar{u}_{0}^{2}-\partial_{2} \bar{u}_{0}^{1} \in H^{\sigma-1}$, with $\sigma-1>1$. Using Estimate (3.17) of Lemma 3.5, we get

$$
\frac{d}{d t}\left\|\bar{u}^{h}(t)\right\|_{\mathbf{H}^{\sigma}}+\gamma\left\|\bar{u}^{h}(t)\right\|_{\mathbf{H}^{\sigma}} \leq C C_{2} e^{-\gamma t}\left\|\bar{u}^{h}(t)\right\|_{\mathbf{H}^{\sigma}},
$$

which leads to

$$
\frac{d}{d t} \ln \left(\left\|\bar{u}^{h}(t)\right\|_{\mathbf{H}^{\sigma}} e^{\gamma t}\right) \leq C C_{2} e^{-\gamma t} .
$$

Integrating this inequality, we obtain

$$
\left\|\bar{u}^{h}(t)\right\|_{\mathbf{H}^{\sigma}} \leq\left(\left\|\bar{u}_{0}^{h}\right\|_{\mathbf{H}^{\sigma}}+\frac{C C_{2}}{\gamma}\right) e^{-\gamma t} .
$$

Now, using Lemma 3.6, we get the following energy estimate in the Sobolev $\mathbf{H}^{\sigma}$-norm for $\bar{u}^{3}$

$$
\frac{1}{2} \frac{d}{d t}\left\|\bar{u}^{3}(t)\right\|_{H^{\sigma}}^{2}+\gamma\left\|\bar{u}^{3}(t)\right\|_{H^{\sigma}}^{2} \leq C\left\|\bar{u}^{h}(t)\right\|_{\mathbf{H}^{\sigma}}\left\|\bar{u}^{3}(t)\right\|_{H^{\sigma}}^{2} .
$$

Using (3.34), we can write

$$
\frac{d}{d t}\left\|\bar{u}^{3}(t)\right\|_{H^{\sigma}}+\gamma\left\|\bar{u}^{3}(t)\right\|_{H^{\sigma}} \leq C\left(\left\|\bar{u}_{0}^{h}\right\|_{\mathbf{H}^{\sigma}}+\frac{C_{2}}{\gamma}\right) e^{-\gamma t}\left\|\bar{u}^{3}(t)\right\|_{H^{\sigma}},
$$

which finally implies that

$$
\left\|\bar{u}^{3}(t)\right\|_{H^{\sigma}} \leq C\left(\left\|\bar{u}_{0}^{3}\right\|_{H^{\sigma}}+\frac{\left\|\bar{u}_{0}^{h}\right\|_{\mathbf{H}^{\sigma}}}{\gamma}+\frac{C_{2}}{\gamma^{2}}\right) e^{-\gamma t} .
$$

Theorem 1.2 is then proved.

Remark 3.7. If $\sigma \in \mathbb{N}$, we do not need Lemma 3.6. Indeed, Theorem 1.2 can be immediately deduced from Lemma 3.5 using the identity

$$
\left\|\nabla_{h}^{\alpha} \nabla_{h} \bar{u}_{h}\right\|_{L^{2}}=\left\|\nabla_{h}^{\alpha} \bar{w}\right\|_{L^{2}}
$$

which holds for all $\alpha \in \mathbb{N}^{2}$.

\section{Proof of the MAIN RESUlt}

In this paragraph, we provide the needed a priori estimates and a sketch of the proof of Theorem 1.1. These a priori estimates can be justified by a classical approximation by smooth fonctions (see for instance [9]). We recall that it is already known that convergence in $\mathbf{L}_{\text {loc }}^{\infty}\left(\mathbb{R}_{+}, \mathbf{L}^{2}\right)$ holds, as proved in $[16]$ and [8]. The important point here is to prove global convergence in time. 
4.1. The corrective boundary layers. Let $\bar{u}$ be the solution of the limiting system (1.3), the third component $\bar{u}^{3}=0$ and we set $\operatorname{curl} \bar{u}^{h}=\partial_{1} \bar{u}^{2}-\partial_{2} \bar{u}^{1}$. Then, as in [16], we can write the boundary layer part in the following form

$$
\mathcal{B}=\mathcal{B}_{1}+\mathcal{B}_{2}+\mathcal{B}_{3}+\mathcal{B}_{4},
$$

where $\mathcal{B}_{i}, i \in\{1,2,3,4\}$, are defined as described below:

1. The term $\mathcal{B}_{1}$ is the main boundary layer term (see (A.7), (A.8) and (A.9) in the appendix)

$$
\mathcal{B}_{1}=\left(\begin{array}{c}
-e^{-\frac{x_{3}}{\varepsilon \sqrt{2 \beta}}}\left(\bar{u}^{1} \cos \frac{x_{3}}{\varepsilon \sqrt{2 \beta}}+\bar{u}^{2} \sin \frac{x_{3}}{\varepsilon \sqrt{2 \beta}}\right)-e^{-\frac{1-x_{3}}{\varepsilon \sqrt{2 \beta}}}\left(\bar{u}^{1} \cos \frac{1-x_{3}}{\varepsilon \sqrt{2 \beta}}+\bar{u}^{2} \sin \frac{1-x_{3}}{\varepsilon \sqrt{2 \beta}}\right) \\
-e^{-\frac{x_{3}}{\varepsilon \sqrt{2 \beta}}}\left(\bar{u}^{2} \cos \frac{x_{3}}{\varepsilon \sqrt{2 \beta}}-\bar{u}^{1} \sin \frac{x_{3}}{\varepsilon \sqrt{2 \beta}}\right)-e^{-\frac{1-x_{3}}{\varepsilon \sqrt{2 \beta}}}\left(\bar{u}^{2} \cos \frac{1-x_{3}}{\varepsilon \sqrt{2 \beta}}-\bar{u}^{1} \sin \frac{1-x_{3}}{\varepsilon \sqrt{2 \beta}}\right) \\
\varepsilon \sqrt{\beta} G\left(x_{3}\right) \operatorname{curl} \bar{u}^{h}
\end{array}\right)
$$

where

$$
G\left(x_{3}\right)=-e^{-\frac{x_{3}}{\varepsilon \sqrt{2 \beta}}} \sin \left(\frac{x_{3}}{\varepsilon \sqrt{2 \beta}}+\frac{\pi}{4}\right)+e^{-\frac{1-x_{3}}{\varepsilon \sqrt{2 \beta}}} \sin \left(\frac{1-x_{3}}{\varepsilon \sqrt{2 \beta}}+\frac{\pi}{4}\right) .
$$

2. The corrective terms $\mathcal{B}_{2}$ and $\mathcal{B}_{3}$ are added to $\mathcal{B}_{1}$ to ensure the Dirichlet boundary conditions at $\left\{x_{3}=0\right\}$ and $\left\{x_{3}=1\right\}$, and we have

$$
\begin{aligned}
& \mathcal{B}_{2}=\left(\begin{array}{c}
\varepsilon \sqrt{2 \beta} \bar{u}^{2} \\
-\varepsilon \sqrt{2 \beta} \bar{u}^{1} \\
\varepsilon \sqrt{2 \beta}\left(\frac{1}{2}-x_{3}\right) \operatorname{curl} \bar{u}^{h}
\end{array}\right) \\
& \mathcal{B}_{3}=e^{-\frac{1}{\varepsilon \sqrt{2 \beta}}} \cos \left(\frac{1}{\varepsilon \sqrt{2 \beta}}\right)\left(\begin{array}{c}
\bar{u}^{1} \\
\bar{u}^{2} \\
0
\end{array}\right) .
\end{aligned}
$$

3. Finally, the term $\mathcal{B}_{4}$ is added to the sum $\mathcal{B}_{1}+\mathcal{B}_{2}+\mathcal{B}_{3}$ to ensure the divergence-free property of $\mathcal{B}$,

$$
\mathcal{B}_{4}=f\left(x_{3}\right)\left(\begin{array}{c}
\bar{u}^{2} \\
-\bar{u}^{1} \\
0
\end{array}\right)+g\left(x_{3}\right)\left(\begin{array}{c}
0 \\
0 \\
\operatorname{curl} \bar{u}^{h}
\end{array}\right)
$$

where

$$
\begin{aligned}
& f\left(x_{3}\right)=a\left[e^{-\frac{x_{3}}{\varepsilon \sqrt{2 \beta}}}+e^{-\frac{1-x_{3}}{\varepsilon \sqrt{2 \beta}}}\right]+b, \\
& g\left(x_{3}\right)=-\varepsilon \sqrt{\beta} e^{-\frac{1}{\varepsilon \sqrt{2 \beta}}} \sin \left(\frac{1}{\varepsilon \sqrt{2 \beta}}+\frac{\pi}{4}\right)-\int_{0}^{x_{3}} f(s) d s,
\end{aligned}
$$

and where $(a, b)$ is the solution of the linear system

$$
\left\{\begin{aligned}
\left(1+e^{-\frac{1}{\varepsilon \sqrt{2 \beta}}}\right) a+b & =-\varepsilon \sqrt{2 \beta}+e^{-\frac{1}{\varepsilon \sqrt{2 \beta}}} \sin \frac{1}{\varepsilon \sqrt{2 \beta}} \\
2 \varepsilon \sqrt{2 \beta}\left(1-e^{-\frac{1}{\varepsilon \sqrt{2 \beta}}}\right) a+b & =2 \varepsilon \sqrt{\beta} e^{-\frac{1}{\varepsilon \sqrt{2 \beta}}} \sin \left(\frac{1}{\varepsilon \sqrt{2 \beta}}+\frac{\pi}{4}\right) .
\end{aligned}\right.
$$

We remark that the determinant of the system (4.7) is

$$
D=1+e^{-\frac{1}{\varepsilon \sqrt{2 \beta}}}-2 \varepsilon \sqrt{2 \beta}\left(1-e^{-\frac{1}{\varepsilon \sqrt{2 \beta}}}\right) .
$$

Thus, for $\varepsilon>0$ small enough, we have $D>\frac{1}{2}$ and (4.7) always has the following solution

$$
a=\frac{J_{\varepsilon}-K_{\varepsilon}}{D} \quad \text { and } \quad b=\frac{K_{\varepsilon}\left(1+e^{-\frac{1}{\varepsilon \sqrt{2 \beta}}}\right)-2 J_{\varepsilon} \varepsilon \sqrt{2 \beta}\left(1-e^{-\frac{1}{\varepsilon \sqrt{2 \beta}}}\right)}{D},
$$

where

$$
\begin{aligned}
J_{\varepsilon} & =-\varepsilon \sqrt{2 \beta}+e^{-\frac{1}{\varepsilon \sqrt{2 \beta}}} \sin \frac{1}{\varepsilon \sqrt{2 \beta}}, \\
K_{\varepsilon} & =2 \varepsilon \sqrt{\beta} e^{-\frac{1}{\varepsilon \sqrt{2 \beta}} \sin \left(\frac{1}{\varepsilon \sqrt{2 \beta}}+\frac{\pi}{4}\right) .}
\end{aligned}
$$

It is easy to prove that if $\varepsilon>0$ is small enough, then

$$
|a|<4(\beta+\sqrt{\beta}) \varepsilon \text { and }|b|<32 \beta \varepsilon^{2} .
$$


With the previously defined boundary layer term $\mathcal{B}$, we can verify that

$$
\operatorname{div}(\bar{u}+\mathcal{B})=0 \quad \text { and } \quad(\bar{u}+\mathcal{B})_{\left.\right|_{\left\{x_{3}=0\right\}}}=(\bar{u}+\mathcal{B})_{\left.\right|_{\left\{x_{3}=1\right\}}}=0 .
$$

Now, let

$$
B_{0}\left(x_{3}\right)=\left[\begin{array}{cc}
-\mathrm{e}^{-\frac{x_{3}}{\varepsilon \sqrt{2 \beta}}} \cos \frac{x_{3}}{\varepsilon \sqrt{2 \beta}}-\mathrm{e}^{-\frac{1-x_{3}}{\varepsilon \sqrt{2 \beta}}} \cos \frac{1-x_{3}}{\varepsilon \sqrt{2 \beta}} & -\mathrm{e}^{-\frac{x_{3}}{\varepsilon \sqrt{2 \beta}}} \sin \frac{x_{3}}{\varepsilon \sqrt{2 \beta}}-\mathrm{e}^{-\frac{1-x_{3}}{\varepsilon \sqrt{2 \beta}}} \sin \frac{1-x_{3}}{\varepsilon \sqrt{2 \beta}} \\
\mathrm{e}^{-\frac{x_{3}}{\varepsilon \sqrt{2 \beta}}} \sin \frac{x_{3}}{\varepsilon \sqrt{2 \beta}}+\mathrm{e}^{-\frac{1-x_{3}}{\varepsilon \sqrt{2 \beta}}} \sin \frac{1-x_{3}}{\varepsilon \sqrt{2 \beta}} & -\mathrm{e}^{-\frac{x_{3}}{\varepsilon \sqrt{2 \beta}}} \cos \frac{x_{3}}{\varepsilon \sqrt{2 \beta}}-\mathrm{e}^{-\frac{1-x_{3}}{\varepsilon \sqrt{2 \beta}}} \cos \frac{1-x_{3}}{\varepsilon \sqrt{2 \beta}}
\end{array}\right]
$$

Then, we can write $\mathcal{B}$ in the following form

$$
\mathcal{B}=\mathcal{M}\left(x_{3}\right)\left(\begin{array}{c}
\bar{u}^{1} \\
\bar{u}^{2} \\
\operatorname{curl} \bar{u}^{h}
\end{array}\right) .
$$

The matrix $\mathcal{M}\left(x_{3}\right)$ is defined by

$$
\mathcal{M}\left(x_{3}\right)=\left[\begin{array}{cc}
M\left(x_{3}\right) & 0 \\
0 & m\left(x_{3}\right)
\end{array}\right]
$$

where

$$
\begin{aligned}
& M\left(x_{3}\right)=B_{0}\left(x_{3}\right)+\left(\varepsilon \sqrt{2 \beta}+f\left(x_{3}\right)\right)\left[\begin{array}{cc}
0 & 1 \\
-1 & 0
\end{array}\right]+e^{-\frac{1}{\varepsilon \sqrt{2 \beta}}} \cos \frac{1}{\varepsilon \sqrt{2 \beta}}\left[\begin{array}{ll}
1 & 0 \\
0 & 1
\end{array}\right], \\
& m\left(x_{3}\right)=\varepsilon \sqrt{\beta} G\left(x_{3}\right)+\varepsilon \sqrt{2 \beta}\left(\frac{1}{2}-x_{3}\right)+g\left(x_{3}\right) .
\end{aligned}
$$

We can also prove the existence of a constant $C>0$ such that, for any $p \geq 1$, we have

$$
\left\{\begin{array}{c}
\|\mathcal{M}(\cdot)\|_{\mathbf{L}_{x_{3}}^{p}} \leq C \varepsilon^{\frac{1}{p}}, \quad\|\mathcal{M}(\cdot)\|_{\mathbf{L}_{x_{3}}^{\infty}} \leq C, \quad\left\|\mathcal{M}^{\prime}(\cdot)\right\|_{\mathbf{L}_{x_{3}}^{p}} \leq C \varepsilon^{\frac{1}{p}-1}, \\
\|m(\cdot)\|_{L_{x_{3}}^{\infty}} \leq C \varepsilon, \quad\|m(\cdot)\|_{L_{x_{3}}^{p}} \leq C \varepsilon \\
\sup _{x_{3} \in\left[0, \frac{1}{2}\right]}\left|x_{3}^{2} M^{\prime}\left(x_{3}\right)\right| \leq C \varepsilon \quad \text { and } \sup _{x_{3} \in\left[\frac{1}{2}, 1\right]}\left|\left(1-x_{3}\right)^{2} M^{\prime}\left(x_{3}\right)\right| \leq C \varepsilon .
\end{array}\right.
$$

4.2. Convergence to the limiting system. It is easy to prove that $\mathcal{B}$ is small, i.e., $\mathcal{B}$ goes to 0 in $\mathbf{L}^{\infty}\left(\mathbb{R}_{+}, \mathbf{L}^{2}\left(\mathbb{R}_{h}^{2} \times[0,1]\right)\right)$ as $\varepsilon$ goes to 0 . Then, our goal is to prove that $v^{\varepsilon}=u^{\varepsilon}-\bar{u}-\mathcal{B}$ converges to 0 in $\mathbf{L}^{\infty}\left(\mathbb{R}_{+}, \mathbf{L}^{2}\left(\mathbb{R}_{h}^{2} \times[0,1]\right)\right)$ as $\varepsilon$ goes to 0 .

Since a two-dimensional divergence-free vector field (independant of $x_{3}$ ) belongs to the kernel of the operator $\mathbb{P}\left(e_{3} \wedge \cdot\right)$, where $\mathbb{P}$ is the Leray projection of $\mathbf{L}^{2}\left(\mathbb{R}^{3}\right)$ onto the subspace of divergence-free vector fields, $e_{3} \wedge \bar{u}$ is a gradient term. Replacing $u^{\varepsilon}$ by $v^{\varepsilon}+\bar{u}+\mathcal{B}$ in the first equation of the system (1.1), and using the fact that $\bar{u}$ is the solution of the system (1.3) and $\bar{u}^{3} \equiv 0$, we deduce that $v^{\varepsilon}$ satisfies the following equation

$$
\begin{aligned}
\partial_{t} v^{\varepsilon}-\nu_{h}(\varepsilon) \Delta_{h} v^{\varepsilon}-\beta \varepsilon & \partial_{3}^{2} v^{\varepsilon}+u^{\varepsilon} \cdot \nabla v^{\varepsilon}+v^{\varepsilon} \cdot \nabla \mathcal{B}+v^{\varepsilon, h} \cdot \nabla_{h} \bar{u} \\
& +\partial_{t} \mathcal{B}+\mathcal{B} \cdot \nabla \mathcal{B}+\mathcal{B}^{h} \cdot \nabla_{h} \bar{u}+\bar{u}^{h} \cdot \nabla_{h} \mathcal{B}-L_{1}-L_{2}+\frac{e_{3} \wedge v^{\varepsilon}}{\varepsilon}=-\nabla \widetilde{p}^{\varepsilon},
\end{aligned}
$$

where

$$
\begin{aligned}
L_{1} & =\nu_{h}(\varepsilon) \Delta_{h} \bar{u}+\nu_{h}(\varepsilon) \Delta_{h} \mathcal{B}, \\
L_{2} & =\beta \varepsilon \partial_{3}^{2} \mathcal{B}-\frac{e_{3} \wedge \mathcal{B}}{\varepsilon}+\sqrt{2 \beta} \bar{u}, \\
\nabla \widetilde{p}^{\varepsilon} & =\nabla p^{\varepsilon}-\nabla_{h} \bar{p}+\frac{e_{3} \wedge \bar{u}}{\varepsilon} .
\end{aligned}
$$

Taking the $\mathbf{L}^{2}$ scalar product of (4.12) with $v^{\varepsilon}$, then integrating by parts the obtained equation and taking into account the fact that $v^{\varepsilon}$ satisfies the Dirichlet boundary condition, we get

$$
\begin{aligned}
& \frac{1}{2} \frac{d}{d t}\left\|v^{\varepsilon}\right\|_{\mathbf{L}^{2}}^{2}+ \nu_{h}(\varepsilon)\left\|\nabla_{h} v^{\varepsilon}\right\|_{\mathbf{L}^{2}}^{2}+\beta \varepsilon\left\|\partial_{3} v^{\varepsilon}\right\|_{\mathbf{L}^{2}}^{2} \\
&=-\left\langle u^{\varepsilon} \cdot \nabla v^{\varepsilon}, v^{\varepsilon}\right\rangle-\left\langle v^{\varepsilon} \cdot \nabla \mathcal{B}, v^{\varepsilon}\right\rangle-\left\langle v^{\varepsilon, h} \cdot \nabla_{h} \bar{u}, v^{\varepsilon}\right\rangle-\left\langle\partial_{t} \mathcal{B}, v^{\varepsilon}\right\rangle \\
& \quad-\left\langle\mathcal{B} \cdot \nabla \mathcal{B}, v^{\varepsilon}\right\rangle-\left\langle\mathcal{B}^{h} \cdot \nabla_{h} \bar{u}, v^{\varepsilon}\right\rangle-\left\langle\bar{u}^{h} \cdot \nabla_{h} \mathcal{B}, v^{\varepsilon}\right\rangle+\left\langle L_{1}, v^{\varepsilon}\right\rangle+\left\langle L_{2}, v^{\varepsilon}\right\rangle .
\end{aligned}
$$


In what follows, we will separately estimate the nine terms on the right-hand side of (4.13). We recall that we will always denote $\mathcal{B}^{1}, \mathcal{B}^{2}$ and $\mathcal{B}^{2}\left(v^{\varepsilon, 1}, v^{\varepsilon, 2}\right.$ and $v^{\varepsilon, 3}$ respectively) the three components of $\mathcal{B}$ $\left(v^{\varepsilon}\right.$ respectively) and we also write $\mathcal{B}=\left(\mathcal{B}^{h}, \mathcal{B}^{3}\right)$ and $v^{\varepsilon}=\left(v^{\varepsilon, h}, v^{\varepsilon, 3}\right)$.

1. The estimate of the first term is classical, using the divergence-free property of $u^{\varepsilon}$ :

$$
\left\langle u^{\varepsilon} \cdot \nabla v^{\varepsilon}, v^{\varepsilon}\right\rangle=0 .
$$

2. The second term is the most difficult to treat. First, we decompose this term as follows

$$
\left\langle v^{\varepsilon} \cdot \nabla \mathcal{B}, v^{\varepsilon}\right\rangle=\left\langle v^{\varepsilon, h} \cdot \nabla_{h} \mathcal{B}^{h}, v^{\varepsilon, h}\right\rangle+\left\langle v^{\varepsilon, h} \cdot \nabla_{h} \mathcal{B}^{3}, v^{\varepsilon, 3}\right\rangle+\left\langle v^{\varepsilon, 3} \partial_{3} \mathcal{B}^{3}, v^{\varepsilon, 3}\right\rangle+\left\langle v^{\varepsilon, 3} \partial_{3} \mathcal{B}^{h}, v^{\varepsilon, h}\right\rangle .
$$

For the first term on the right-hand side of (4.15), Hölder's inequality implies that

$$
\left|\left\langle v^{\varepsilon, h} \cdot \nabla_{h} \mathcal{B}^{h}, v^{\varepsilon, h}\right\rangle\right| \leq C\left\|v^{\varepsilon, h}\right\|_{\mathbf{L}^{2}}\left\|\nabla_{h} \mathcal{B}^{h}\right\|_{\mathbf{L}^{\infty}}\left\|v^{\varepsilon, h}\right\|_{\mathbf{L}^{2}} \leq C\|\mathcal{M}(\cdot)\|_{\mathbf{L}_{x_{3}}^{\infty}}\left\|\nabla_{h} \bar{u}^{h}\right\|_{\mathbf{L}_{x_{h}}^{\infty}}\left\|v^{\varepsilon}\right\|_{\mathbf{L}^{2}}^{2} .
$$

Then, using Estimates (4.11) and Lemma 3.5, we obtain

$$
\left|\left\langle v^{\varepsilon, h} \cdot \nabla_{h} \mathcal{B}^{h}, v^{\varepsilon, h}\right\rangle\right| \leq C\left(\bar{u}_{0}\right) \mathrm{e}^{-t \sqrt{2 \beta}}\left\|v^{\varepsilon}\right\|_{\mathbf{L}^{2}}^{2} .
$$

Next, using integrations by parts and Hölder's inequality, we deduce that

$$
\left|\left\langle v^{\varepsilon, h} \cdot \nabla_{h} \mathcal{B}^{3}, v^{\varepsilon, 3}\right\rangle\right| \leq C\left\|\nabla_{h} v^{\varepsilon}\right\|_{\mathbf{L}^{2}}\left\|\mathcal{B}^{3}\right\|_{\mathbf{L}^{\infty}}\left\|v^{\varepsilon}\right\|_{\mathbf{L}^{2}} .
$$

So, Estimates (4.11), Lemmas 3.1 and 3.5 and Young's inequality imply

$$
\begin{aligned}
\left|\left\langle v^{\varepsilon, h} \cdot \nabla_{h} \mathcal{B}^{3}, v^{\varepsilon, 3}\right\rangle\right| & \leq C\|m(\cdot)\|_{\mathbf{L}_{x_{3}}^{\infty}}\left\|\operatorname{curl} \bar{u}^{h}\right\|_{\mathbf{L}_{x_{h}}^{\infty}}\left\|\nabla_{h} v^{\varepsilon}\right\|_{\mathbf{L}^{2}}\left\|v^{\varepsilon}\right\|_{\mathbf{L}^{2}} \\
& \leq C\left(\bar{u}_{0}\right) \varepsilon \mathrm{e}^{-t \sqrt{2 \beta}}\left\|v^{\varepsilon}\right\|_{\mathbf{L}^{2}}\left\|\nabla_{h} v^{\varepsilon}\right\|_{\mathbf{L}^{2}} \\
& \leq C\left(\bar{u}_{0}\right) \varepsilon \mathrm{e}^{-t \sqrt{2 \beta}}\left\|v^{\varepsilon}\right\|_{\mathbf{L}^{2}}^{2}+\frac{C\left(\bar{u}_{0}\right) \varepsilon}{8}\left\|\nabla_{h} v^{\varepsilon}\right\|_{\mathbf{L}^{2}}^{2} .
\end{aligned}
$$

Performing an integration by parts, we can control the third term on the right-hand side of (4.15) in the same way as the second one:

$$
\begin{aligned}
\left|\left\langle v^{\varepsilon, 3} \partial_{3} \mathcal{B}^{3}, v^{\varepsilon, 3}\right\rangle\right| & =2\left|\left\langle\mathcal{B}^{3} v^{\varepsilon, 3}, \partial_{3} v^{\varepsilon, 3}\right\rangle\right|=2\left|\left\langle\mathcal{B}^{3} v^{\varepsilon, 3}, \operatorname{div}_{h} v^{\varepsilon, h}\right\rangle\right| \\
& \leq C\left(\bar{u}_{0}\right) \varepsilon \mathrm{e}^{-t \sqrt{2 \beta}}\left\|v^{\varepsilon}\right\|_{\mathbf{L}^{2}}^{2}+\frac{C\left(\bar{u}_{0}\right) \varepsilon}{8}\left\|\nabla_{h} v^{\varepsilon}\right\|_{\mathbf{L}^{2}}^{2}
\end{aligned}
$$

In order to estimate the last term of the right-hand side of (4.15), we decompose it into two parts, the first part corresponding to the boundary layer near $\left\{x_{3}=0\right\}$ and the other part corresponding to the one near $\left\{x_{3}=1\right\}$ :

$$
\left\langle v^{\varepsilon, 3} \partial_{3} \mathcal{B}^{h}, v^{\varepsilon, h}\right\rangle=\int_{\mathbb{R}_{h}^{2} \times\left[0, \frac{1}{2}\right]}\left(v^{\varepsilon, 3} \partial_{3} \mathcal{B}^{h}\right) \cdot v^{\varepsilon, h} d x+\int_{\mathbb{R}_{h}^{2} \times\left[\frac{1}{2}, 1\right]}\left(v^{\varepsilon, 3} \partial_{3} \mathcal{B}^{h}\right) \cdot v^{\varepsilon, h} d x
$$

For the first part, since $v^{\varepsilon}$ vanishes on $\left\{x_{3}=0\right\}$, using Hölder's inequality and Hardy's inequality, we get

$$
\begin{aligned}
\left|\int_{\mathbb{R}_{h}^{2} \times\left[0, \frac{1}{2}\right]}\left(v^{\varepsilon, 3} \partial_{3} \mathcal{B}^{h}\right) \cdot v^{\varepsilon, h} d x\right| & \leq \sup _{x_{3} \in\left[0, \frac{1}{2}\right]}\left|x_{3}^{2} M^{\prime}\left(x_{3}\right)\right|\|\bar{u}\|_{\mathbf{L}_{x_{h}}}\left\|\frac{v^{\varepsilon, 3}}{x_{3}}\right\|_{L^{2}}\left\|\frac{v^{\varepsilon, h}}{x_{3}}\right\|_{\mathbf{L}^{2}} \\
& \leq \sup _{x_{3} \in\left[0, \frac{1}{2}\right]}\left|x_{3}^{2} M^{\prime}\left(x_{3}\right)\right|\|\bar{u}\|_{\mathbf{L}_{x_{h}}^{\infty}}\left\|\partial_{3} v^{\varepsilon, 3}\right\|_{L^{2}}\left\|\partial_{3} v^{\varepsilon, h}\right\|_{\mathbf{L}^{2}} .
\end{aligned}
$$

We recall that $\partial_{3} v^{\varepsilon, 3}=-\operatorname{div}_{h} v^{\varepsilon, h}$. Then, Lemmas 3.1 and 3.5, Estimates (4.11) and Young's inequality imply

$$
\begin{aligned}
\left|\int_{\mathbb{R}_{h}^{2} \times\left[0, \frac{1}{2}\right]}\left(v^{\varepsilon, 3} \partial_{3} \mathcal{B}^{h}\right) \cdot v^{\varepsilon, h} d x\right| & \leq C\left(\bar{u}_{0}\right) \varepsilon \mathrm{e}^{-t \sqrt{2 \beta}}\left\|\operatorname{div}_{h} v^{\varepsilon, 3}\right\|_{\mathbf{L}^{2}}\left\|\partial_{3} v^{\varepsilon, h}\right\|_{\mathbf{L}^{2}} \\
& \leq \frac{C\left(\bar{u}_{0}\right) \varepsilon}{8}\left\|\nabla_{h} v^{\varepsilon}\right\|_{\mathbf{L}^{2}}^{2}+\frac{\beta \varepsilon}{4}\left\|\partial_{3} v^{\varepsilon}\right\|_{\mathbf{L}^{2}}^{2} .
\end{aligned}
$$


For the second part concerning the boundary layer near $\left\{x_{3}=1\right\}$, since $v^{\varepsilon}=\left(v^{\varepsilon, h}, v^{\varepsilon, 3}\right)$ vanishes on $\left\{x_{3}=1\right\}$, Hardy's inequality implies that

$$
\begin{aligned}
I_{v}=\int_{\mathbb{R}_{h}^{2}}\left(\int_{\frac{1}{2}}^{1}\left|\frac{v^{\varepsilon, 3}\left(x_{h}, x_{3}\right)}{1-x_{3}}\right|^{2} d x_{3}\right) d x_{h} & =\int_{\mathbb{R}_{h}^{2}}\left(\int_{0}^{\frac{1}{2}}\left|\frac{v^{\varepsilon, 3}\left(x_{h}, 1-x_{3}\right)}{x_{3}}\right|^{2} d x_{3}\right) d x_{h} \\
& \leq C \int_{\mathbb{R}_{h}^{2}}\left(\int_{0}^{\frac{1}{2}}\left|\partial_{3} v^{\varepsilon, 3}\left(x_{h}, 1-x_{3}\right)\right|^{2} d x_{3}\right) d x_{h} \\
& \leq C\left\|\partial_{3} v^{\varepsilon, 3}\right\|_{L^{2}}^{2}=C\left\|\operatorname{div}_{h} v^{\varepsilon, h}\right\|_{L^{2}}^{2} .
\end{aligned}
$$

Likewise,

$$
I_{h}=\int_{\mathbb{R}_{h}^{2}}\left(\int_{\frac{1}{2}}^{1}\left|\frac{v^{\varepsilon, h}\left(x_{h}, x_{3}\right)}{1-x_{3}}\right|^{2} d x_{3}\right) d x_{h} \leq C\left\|\partial_{3} v^{\varepsilon, h}\right\|_{L^{2}}^{2} .
$$

Thus, using Hölder's inequality, we get

$$
\begin{aligned}
\left|\int_{\mathbb{R}_{h}^{2} \times\left[\frac{1}{2}, 1\right]}\left(v^{\varepsilon, 3} \partial_{3} \mathcal{B}^{h}\right) \cdot v^{\varepsilon, h} d x\right| & \leq \sup _{x_{3} \in\left[\frac{1}{2}, 1\right]}\left|\left(1-x_{3}\right)^{2} M^{\prime}\left(x_{3}\right)\right|\|\bar{u}\|_{\mathbf{L}_{x_{h}}^{\infty}} \sqrt{I_{v}} \sqrt{I_{h}} \\
& \leq C\left(\bar{u}_{0}\right) \varepsilon \mathrm{e}^{-t \sqrt{2 \beta}}\left\|\operatorname{div}_{h} v^{\varepsilon, 3}\right\|_{\mathbf{L}^{2}}\left\|\partial_{3} v^{\varepsilon, h}\right\|_{\mathbf{L}^{2}} \\
& \leq \frac{C\left(\bar{u}_{0}\right) \varepsilon}{8}\left\|\nabla_{h} v^{\varepsilon}\right\|_{\mathbf{L}^{2}}^{2}+\frac{\beta \varepsilon}{4}\left\|\partial_{3} v^{\varepsilon}\right\|_{\mathbf{L}^{2}}^{2} .
\end{aligned}
$$

3. The third term on the right-hand side of (4.13) can be treated using Hölder's inequality and Lemma 3.5. We have

$$
\left|\left\langle v^{\varepsilon, h} \cdot \nabla_{h} \bar{u}, v^{\varepsilon}\right\rangle\right| \leq C\left\|\nabla_{h} \bar{u}^{h}\right\|_{\mathbf{L}^{\infty}\left(\mathbb{R}_{h}^{2}\right)}\left\|v^{\varepsilon}\right\|_{\mathbf{L}^{2}}^{2} \leq C\left(\bar{u}_{0}\right) \mathrm{e}^{-t \sqrt{2 \beta}}\left\|v^{\varepsilon}\right\|_{\mathbf{L}^{2}}^{2} .
$$

4. We recall that

$$
\mathcal{B}=\mathcal{M}\left(x_{3}\right){ }^{t} A=\left[\begin{array}{cc}
M\left(x_{3}\right) & 0 \\
0 & m\left(x_{3}\right)
\end{array}\right]\left(\begin{array}{c}
\bar{u}^{1} \\
\bar{u}^{2} \\
\operatorname{curl} \bar{u}^{h}
\end{array}\right)
$$

Then, we can write the fourth term as

$$
\left\langle\partial_{t} \mathcal{B}, v^{\varepsilon}\right\rangle=\left\langle M\left(x_{3}\right) \partial_{t} \bar{u}^{h}, v^{\varepsilon, h}\right\rangle+\left\langle m\left(x_{3}\right) \partial_{t} \operatorname{curl} \bar{u}^{h}, v^{\varepsilon, 3}\right\rangle .
$$

Let $\mathbb{P}_{\mathbb{R}_{h}^{2}}$ be the Leray projection of $\mathbf{L}^{2}\left(\mathbb{R}_{h}^{2}\right)$ onto the subspace of two dimensional divergence-free vector fields. Since $\bar{u}^{3}=0$, and $\partial_{3} \bar{p}=0$, using Estimate (4.11) and Lemmas 3.1 and 3.5, we have

$$
\begin{aligned}
\left|\left\langle M\left(x_{3}\right) \partial_{t} \bar{u}^{h}, v^{\varepsilon, h}\right\rangle\right| & \leq\left|\left\langle M\left(x_{3}\right) \mathbb{P}_{\mathbb{R}_{h}^{2}}\left(\bar{u}^{h} \cdot \nabla_{h} \bar{u}^{h}\right), v^{\varepsilon, h}\right\rangle\right|+\sqrt{2 \beta}\left|\left\langle M\left(x_{3}\right) \bar{u}^{h}, v^{\varepsilon, h}\right\rangle\right| \\
& \leq\|M(\cdot)\|_{\mathbf{L}_{x_{3}}^{2}}\left(\left\|\mathbb{P}_{\mathbb{R}_{h}^{2}}\left(\bar{u}^{h} \cdot \nabla_{h} \bar{u}^{h}\right)\right\|_{\mathbf{L}^{2}}+\sqrt{2 \beta}\left\|\bar{u}^{h}\right\|_{\mathbf{L}^{2}}\right)\left\|v^{\varepsilon, h}\right\|_{\mathbf{L}^{2}} \\
& \leq C \varepsilon^{\frac{1}{2}}\left\|\bar{u}^{h}\right\|_{\mathbf{L}^{2}}\left(1+\left\|\nabla_{h} \bar{u}^{h}\right\|_{\mathbf{L}^{\infty}}\right)\left\|v^{\varepsilon}\right\|_{\mathbf{L}^{2}} \\
& \leq C\left(\bar{u}_{0}\right) \varepsilon^{\frac{1}{2}} \mathrm{e}^{-t \sqrt{2 \beta}}\left(1+\left\|v^{\varepsilon}\right\|_{\mathbf{L}^{2}}^{2}\right) .
\end{aligned}
$$

Applying the operator curl to the first two equations of the system (1.3) (we recall that in this paper, curl only acts on the horizontal components and we already defined $\operatorname{curl} \bar{u}^{h}=\partial_{1} \bar{u}^{2}-\partial_{2} \bar{u}^{1}$ ), we obtain

$$
\partial_{t}\left(\operatorname{curl} \bar{u}^{h}\right)+\bar{u}^{h} \cdot \nabla_{h}\left(\operatorname{curl} \bar{u}^{h}\right)+\sqrt{2 \beta}\left(\operatorname{curl} \bar{u}^{h}\right)=0 .
$$

Then,

$$
\begin{aligned}
\left|\left\langle m\left(x_{3}\right) \partial_{t} \operatorname{curl} \bar{u}^{h}, v^{\varepsilon, 3}\right\rangle\right| & \leq\left|\left\langle m\left(x_{3}\right)^{h} \cdot \nabla_{h} \operatorname{curl} \bar{u}^{h}, v^{\varepsilon, 3}\right\rangle\right|+\sqrt{2 \beta}\left|\left\langle m\left(x_{3}\right) \operatorname{curl} \bar{u}^{h}, v^{\varepsilon, 3}\right\rangle\right| \\
& \leq\|m(\cdot)\|_{\mathbf{L}_{x_{3}}^{2}}\left(\left\|\bar{u}^{h} \cdot \nabla_{h} \operatorname{curl} \bar{u}^{h}\right\|_{\mathbf{L}^{2}}+\sqrt{2 \beta}\left\|\operatorname{curl} \bar{u}^{h}\right\|_{\mathbf{L}^{2}}\right)\left\|v^{\varepsilon, 3}\right\|_{\mathbf{L}^{2}} \\
& \leq C \varepsilon\left(\left\|\bar{u}^{h}\right\|_{\mathbf{L} \infty}\left\|\nabla_{h} \operatorname{curl} \bar{u}^{h}\right\|_{\mathbf{L}^{2}}+\left\|\operatorname{curl} \bar{u}^{h}\right\|_{\mathbf{L}^{2}}\right)\left\|v^{\varepsilon}\right\|_{\mathbf{L}^{2}} \\
& \leq C\left(\bar{u}_{0}\right) \varepsilon \mathrm{e}^{-t \sqrt{2 \beta}}\left(1+\left\|v^{\varepsilon}\right\|_{\mathbf{L}^{2}}^{2}\right) .
\end{aligned}
$$

5. We decompose the fifth term into two parts:

$$
\left\langle\mathcal{B} \cdot \nabla \mathcal{B}, v^{\varepsilon}\right\rangle=\left\langle\mathcal{B}^{h} \cdot \nabla_{h} \mathcal{B}, v^{\varepsilon}\right\rangle+\left\langle\mathcal{B}^{3} \partial_{3} \mathcal{B}, v^{\varepsilon}\right\rangle .
$$


The "horizontal" part can be bounded using Hölder's inequality, Estimates (4.11) and Theorem 1.2 as follows

$$
\begin{aligned}
\left|\left\langle\mathcal{B}^{h} \cdot \nabla_{h} \mathcal{B}, v^{\varepsilon}\right\rangle\right| & \leq C\left\|\mathcal{B}^{h}\right\|_{\mathbf{L}^{\infty}}\left\|\nabla_{h} \mathcal{B}\right\|_{\mathbf{L}^{2}}\left\|v^{\varepsilon}\right\|_{\mathbf{L}^{2}} \\
& \leq C\|M(\cdot)\|_{\mathbf{L}_{x_{3}}^{\infty}}\left\|\bar{u}^{h}\right\|_{\mathbf{L}^{\infty}}\|\mathcal{M}(\cdot)\|_{\mathbf{L}_{x_{3}}^{2}}\left(\left\|\nabla_{h} \bar{u}^{h}\right\|_{\mathbf{L}^{2}}+\left\|\nabla_{h} \operatorname{curl} \bar{u}^{h}\right\|_{\mathbf{L}^{2}}\right)\left\|v^{\varepsilon}\right\|_{\mathbf{L}^{2}} \\
& \leq C \varepsilon^{\frac{1}{2}}\left\|\bar{u}^{h}\right\|_{\mathbf{H}^{\sigma}}^{2}\left\|v^{\varepsilon}\right\|_{\mathbf{L}^{2}} \leq C\left(\bar{u}_{0}\right) \varepsilon^{\frac{1}{2}} e^{-t \sqrt{2 \beta}}\left(1+\left\|v^{\varepsilon}\right\|_{\mathbf{L}^{2}}^{2}\right) .
\end{aligned}
$$

Likewise, we have the following estimate for the "vertical" part:

$$
\begin{aligned}
\left|\left\langle\mathcal{B}^{3} \partial_{3} \mathcal{B}, v^{\varepsilon}\right\rangle\right| & \leq\left\|\mathcal{B}^{3}\right\|_{L^{\infty}}\left\|\partial_{3} \mathcal{B}\right\|_{\mathbf{L}^{2}}\left\|v^{\varepsilon}\right\|_{\mathbf{L}^{2}} \\
& \leq C\left(\bar{u}_{0}\right) \mathrm{e}^{-t \sqrt{2 \beta}}\|m(\cdot)\|_{L_{x_{3}}^{\infty}}\left\|\mathcal{M}^{\prime}(\cdot)\right\|_{\mathbf{L}_{x_{3}}^{2}}\left\|v^{\varepsilon}\right\|_{\mathbf{L}^{2}} \\
& \leq C\left(\bar{u}_{0}\right) \varepsilon^{\frac{1}{2}} \mathrm{e}^{-t \sqrt{2 \beta}}\left(1+\left\|v^{\varepsilon}\right\|_{\mathbf{L}^{2}}^{2}\right) .
\end{aligned}
$$

6. For the sixth term, taking into account the fact that $\bar{u}$ is independent of $x_{3}$, Estimates (4.11) and Lemma 3.1 imply

$$
\begin{aligned}
\left|\left\langle\mathcal{B}^{h} \cdot \nabla_{h} \bar{u}, v^{\varepsilon}\right\rangle\right| & \leq\left\|\mathcal{B}^{h}\right\|_{\mathbf{L}_{x_{h}}^{4} \mathbf{L}_{x_{3}}^{2}}\left\|\nabla_{h} \bar{u}\right\|_{\mathbf{L}_{x_{h}}^{4}}\left\|v^{\varepsilon}\right\|_{\mathbf{L}^{2}} \\
& \leq\|M(\cdot)\|_{\mathbf{L}_{x_{3}}^{2}}\left(\|\bar{u}\|_{\mathbf{L}^{4}}^{2}+\left\|\nabla_{h} \bar{u}\right\|_{\mathbf{L}^{4}}^{2}\right)\left\|v^{\varepsilon}\right\|_{\mathbf{L}^{2}} \\
& \leq C\left(\bar{u}_{0}\right) \varepsilon^{\frac{1}{2}} \mathrm{e}^{-t \sqrt{2 \beta}}\left(1+\left\|v^{\varepsilon}\right\|_{\mathbf{L}^{2}}^{2}\right) .
\end{aligned}
$$

7. The seventh term can be bounded in a similar way as the sixth one.

$$
\begin{aligned}
\left|\left\langle\bar{u}^{h} \cdot \nabla_{h} \mathcal{B}, v^{\varepsilon}\right\rangle\right| & \leq\left\|\bar{u}^{h}\right\|_{\mathbf{L}^{\infty}}\left\|\nabla_{h} \mathcal{B}\right\|_{\mathbf{L}^{2}}\left\|v^{\varepsilon}\right\|_{\mathbf{L}^{2}} \\
& \leq C\left\|\bar{u}^{h}\right\|_{\mathbf{L}^{\infty}}\|\mathcal{M}(\cdot)\|_{\mathbf{L}_{x_{3}}^{2}}\left(\left\|\nabla_{h} \bar{u}^{h}\right\|_{\mathbf{L}^{2}}+\left\|\nabla_{h} \operatorname{curl} \bar{u}^{h}\right\|_{\mathbf{L}^{2}}\right)\left\|v^{\varepsilon}\right\|_{\mathbf{L}^{2}} \\
& \leq C\left(\bar{u}_{0}\right) \varepsilon^{\frac{1}{2}} \mathrm{e}^{-t \sqrt{2 \beta}}\left(1+\left\|v^{\varepsilon}\right\|_{\mathbf{L}^{2}}^{2}\right) .
\end{aligned}
$$

8. We write the eighth term as

$$
\left\langle L_{1}, v^{\varepsilon}\right\rangle=\nu_{h}(\varepsilon)\left\langle\Delta_{h} \bar{u}^{h}, v^{\varepsilon, h}\right\rangle+\nu_{h}(\varepsilon)\left\langle\Delta_{h} \mathcal{B}, v^{\varepsilon}\right\rangle .
$$

Then, we have

$$
\begin{aligned}
\left|\nu_{h}(\varepsilon)\left\langle\Delta_{h} \bar{u}^{h}, v^{\varepsilon, h}\right\rangle\right| & \leq \nu_{h}(\varepsilon)\left\|\bar{u}^{h}\right\|_{\mathbf{H}^{\sigma}}\left\|v^{\varepsilon}\right\|_{\mathbf{L}^{2}} \\
& \leq C\left(\bar{u}_{0}\right) \nu_{h}(\varepsilon) e^{-t \sqrt{2 \beta}}\left(1+\left\|v^{\varepsilon}\right\|_{\mathbf{L}^{2}}^{2}\right)
\end{aligned}
$$

and

$$
\begin{aligned}
\left|\nu_{h}(\varepsilon)\left\langle\Delta_{h} \mathcal{B}, v^{\varepsilon}\right\rangle\right| & =\nu_{h}(\varepsilon)\left|\left\langle\nabla_{h} \mathcal{B}, \nabla_{h} v^{\varepsilon}\right\rangle\right| \leq \nu_{h}(\varepsilon)\left\|\nabla_{h} \mathcal{B}\right\|_{\mathbf{L}^{2}}\left\|\nabla_{h} v^{\varepsilon}\right\|_{\mathbf{L}^{2}} \\
& \leq \nu_{h}(\varepsilon)\|\mathcal{M}(\cdot)\|_{\mathbf{L}_{x_{3}}^{2}}\left(\left\|\nabla_{h} \bar{u}^{h}\right\|_{\mathbf{L}^{2}}+\left\|\nabla_{h} \operatorname{curl} \bar{u}^{h}\right\|_{\mathbf{L}^{2}}\right)\left\|v^{\varepsilon}\right\|_{\mathbf{L}^{2}} \\
& \leq C\left(\bar{u}_{0}\right) \nu_{h}(\varepsilon) \varepsilon^{\frac{1}{2}} e^{-t \sqrt{2 \beta}}\left(1+\left\|v^{\varepsilon}\right\|_{\mathbf{L}^{2}}^{2}\right) .
\end{aligned}
$$

9. We will estimate the ninth term as in [16] or [21]. We have

$$
\left\langle L_{2}, v^{\varepsilon}\right\rangle=\left\langle\beta \varepsilon \partial_{3}^{2} \mathcal{B}, v^{\varepsilon}\right\rangle-\left\langle\frac{e_{3} \wedge \mathcal{B}}{\varepsilon}, v^{\varepsilon}\right\rangle+\left\langle\sqrt{2 \beta} \bar{u}, v^{\varepsilon}\right\rangle .
$$

We recall the definition $(4.1)$ of $\mathcal{B}$

$$
\mathcal{B}=\mathcal{B}_{1}+\mathcal{B}_{2}+\mathcal{B}_{3}+\mathcal{B}_{4},
$$

and for any $i \in\{1,2,3,4\}$, we set $\mathcal{B}_{i}=\left(\mathcal{B}_{i}^{h}, \mathcal{B}_{i}^{3}\right)$, where $\mathcal{B}_{i}^{h}$ and $\mathcal{B}_{i}^{3}$ denote the horizontal and vertical components of $\mathcal{B}_{i}$ respectively. We also recall that $\mathcal{B}=\left(\mathcal{B}^{h}, \mathcal{B}^{3}\right)$. Then, the following identities are immediate

$$
\begin{gathered}
\partial_{3}^{2} \mathcal{B}_{3}^{h}=0, \\
\beta \varepsilon \partial_{3}^{2} \mathcal{B}_{1}^{h}-\frac{e_{3} \wedge \mathcal{B}_{1}}{\varepsilon}=0, \\
\beta \varepsilon \partial_{3}^{2} \mathcal{B}_{2}^{h}-\frac{e_{3} \wedge \mathcal{B}_{2}}{\varepsilon}+\sqrt{2 \beta} \bar{u}=0 .
\end{gathered}
$$


For the remaining terms, we have

$$
\begin{aligned}
& \beta \varepsilon\left|\left\langle\partial_{3}^{2} \mathcal{B}^{3}, v^{\varepsilon, 3}\right\rangle\right| \leq \beta \varepsilon\left\|\partial_{3} \mathcal{B}^{3}\right\|_{\mathbf{L}^{2}}\left\|\partial_{3} v^{\varepsilon, 3}\right\| \\
& \leq \beta \varepsilon\left\|\nabla_{h} \mathcal{B}^{h}\right\|_{\mathbf{L}^{2}}\left\|\nabla_{h} v^{\varepsilon}\right\|_{\mathbf{L}^{2}} \\
& \leq \beta \varepsilon\left\|M\left(x_{3}\right)\right\|_{\mathbf{L}_{x_{3}}^{2}}\left\|\nabla_{h} \bar{u}^{h}\right\|_{\mathbf{L}_{x_{h}}^{2}}\left\|\nabla_{h} v^{\varepsilon}\right\|_{\mathbf{L}^{2}} \\
& \leq C\left(\bar{u}_{0}\right) \varepsilon^{2} \mathrm{e}^{-t \sqrt{2 \beta}}+\frac{C\left(\bar{u}_{0}\right) \varepsilon}{8}\left\|\nabla_{h} v^{\varepsilon}\right\|_{\mathbf{L}^{2}}^{2} ; \\
&\left|\left\langle\frac{e_{3} \wedge \mathcal{B}_{3}}{\varepsilon}, v^{\varepsilon, h}\right\rangle\right| \leq C \varepsilon^{-1} \mathrm{e}^{-\frac{1}{\varepsilon}}\|\bar{u}\|_{\mathbf{L}^{2}}\left\|v^{\varepsilon}\right\|_{\mathbf{L}^{2}} \leq C\left(\bar{u}_{0}\right) \varepsilon^{\frac{1}{2}} \mathrm{e}^{-t \sqrt{2 \beta}}\left(1+\left\|v^{\varepsilon}\right\|_{\mathbf{L}^{2}}^{2}\right) .
\end{aligned}
$$

We recall that

where $f$ is defined in (4.6),

$$
\mathcal{B}_{4}^{h}=f\left(x_{3}\right)\left(\begin{array}{c}
\bar{u}^{2} \\
-\bar{u}^{1}
\end{array}\right)
$$

$$
f\left(x_{3}\right)=a\left[\exp \left(-\frac{x_{3}}{\varepsilon \sqrt{2 \beta}}\right)+\exp \left(-\frac{1-x_{3}}{\varepsilon \sqrt{2 \beta}}\right)\right]+b
$$

and that, if $\varepsilon>0$ is small enough, we have

$$
|a|<4(\beta+\sqrt{\beta}) \varepsilon \text { and }|b|<32 \beta \varepsilon^{2} .
$$

Then,

$$
\begin{aligned}
\beta \varepsilon\left|\left\langle\partial_{3}^{2} \mathcal{B}_{4}^{h}, v^{\varepsilon, h}\right\rangle\right| & \leq \beta \varepsilon\left\|f^{\prime \prime}(\cdot)\right\|_{L_{x_{3}}^{2}}\left\|\bar{u}^{h}\right\|_{\mathbf{L}^{2}}\left\|v^{\varepsilon, h}\right\|_{\mathbf{L}^{2}} \\
& \leq C \varepsilon^{\frac{1}{2}}\left\|\bar{u}^{h}\right\|_{\mathbf{L}^{2}}\left\|v^{\varepsilon}\right\|_{\mathbf{L}^{2}} \\
& \leq C\left(\bar{u}_{0}\right) \varepsilon^{\frac{1}{2}} \mathrm{e}^{-t \sqrt{2 \beta}}\left(1+\left\|v^{\varepsilon}\right\|_{\mathbf{L}^{2}}^{2}\right) .
\end{aligned}
$$

Finally, we have

$$
\begin{aligned}
\left|\left\langle\frac{e_{3} \wedge \mathcal{B}_{4}}{\varepsilon}, v^{\varepsilon, h}\right\rangle\right| & \leq C\left[\left(\int_{0}^{1}\left|\mathrm{e}^{-\frac{x_{3}}{\varepsilon \sqrt{2 \beta}}}+\mathrm{e}^{-\frac{1-x_{3}}{\varepsilon \sqrt{2 \beta}}}\right|^{2} d x_{3}\right)^{\frac{1}{2}}+\beta \varepsilon\right]\left\|\bar{u}^{h}\right\|_{\mathbf{L}^{2}}\left\|v^{\varepsilon, h}\right\|_{\mathbf{L}^{2}} \\
& \leq C\left(\bar{u}_{0}\right) \varepsilon^{\frac{1}{2}} \mathrm{e}^{-t \sqrt{2 \beta}}\left(1+\left\|v^{\varepsilon}\right\|_{\mathbf{L}^{2}}^{2}\right) .
\end{aligned}
$$

End of the proof of Theorem 1.1: Summing all the inequalities from (4.14) to (4.33), we deduce from (4.13) that, for $\varepsilon>0$ small enough

$$
\begin{aligned}
& \frac{d}{d t}\left\|v^{\varepsilon}\right\|_{\mathbf{L}^{2}}^{2}+\nu_{h}(\varepsilon)\left\|\nabla_{h} v^{\varepsilon}\right\|_{\mathbf{L}^{2}}^{2}+\beta \varepsilon\left\|\partial_{3} v^{\varepsilon}\right\|_{\mathbf{L}^{2}}^{2} \\
& \quad \leq C\left(\bar{u}_{0}\right)\left(\varepsilon^{\frac{1}{2}}+\nu_{h}(\varepsilon)\right) \mathrm{e}^{-t \sqrt{2 \beta}}+C\left(\bar{u}_{0}\right) \mathrm{e}^{-t \sqrt{2 \beta}}\left\|v^{\varepsilon}\right\|_{\mathbf{L}^{2}}^{2}+C\left(\bar{u}_{0}\right) \varepsilon\left\|\nabla_{h} v^{\varepsilon}\right\|_{\mathbf{L}^{2}}^{2} .
\end{aligned}
$$

If $C\left(\bar{u}_{0}\right) \varepsilon \leq \nu_{h}(\varepsilon)$, for any $\varepsilon>0$ small enough, by integrating the above inequality with respect to the time variable, we get

$$
\left\|v^{\varepsilon}(t)\right\|_{\mathbf{L}^{2}}^{2} \leq\left\|v^{\varepsilon}(0)\right\|_{\mathbf{L}^{2}}^{2}+C\left(\bar{u}_{0}\right) \frac{\varepsilon^{\frac{1}{2}}+\nu_{h}(\varepsilon)}{\sqrt{2 \beta}}+C\left(\bar{u}_{0}\right) \int_{0}^{t} \mathrm{e}^{-s \sqrt{2 \beta}}\left\|v^{\varepsilon}(s)\right\|_{\mathbf{L}^{2}}^{2} d s .
$$

We recall that $v^{\varepsilon}=u^{\varepsilon}-\bar{u}-\mathcal{B}$. Thus,

$$
\begin{aligned}
\left\|v^{\varepsilon}(0)\right\|_{\mathbf{L}^{2}}^{2} & \leq\left\|u^{\varepsilon}(0)-\bar{u}(0)\right\|_{\mathbf{L}^{2}}^{2}+\|\mathcal{B}(0)\|_{\mathbf{L}^{2}}^{2} \\
& \leq\left\|u_{0}^{\varepsilon}-\bar{u}_{0}\right\|_{\mathbf{L}^{2}}^{2}+\|\mathcal{M}(\cdot)\|_{\mathbf{L}_{x_{3}}^{2}}^{2}\left\|\bar{u}_{0}\right\|_{\mathbf{L}^{2}}^{2} \leq\left\|u_{0}^{\varepsilon}-\bar{u}_{0}\right\|_{\mathbf{L}^{2}}^{2}+C \varepsilon^{\frac{1}{2}}\left\|\bar{u}_{0}\right\|_{\mathbf{L}^{2}}^{2} .
\end{aligned}
$$

According to the Gronwall lemma, it follows from (4.34) that

$$
\left\|v^{\varepsilon}(t)\right\|_{\mathbf{L}^{2}}^{2} \leq\left(\left\|u_{0}^{\varepsilon}-\bar{u}_{0}\right\|_{\mathbf{L}^{2}}^{2}+C \varepsilon^{\frac{1}{2}}\left\|\bar{u}_{0}\right\|_{\mathbf{L}^{2}}^{2}+C\left(\bar{u}_{0}\right) \frac{\varepsilon^{\frac{1}{2}}+\nu_{h}(\varepsilon)}{\sqrt{2 \beta}}\right) \exp \left\{\frac{C\left(\bar{u}_{0}\right)}{\sqrt{2 \beta}}\right\},
$$

which leads to

$$
\lim _{\varepsilon \rightarrow 0}\left\|v^{\varepsilon}\right\|_{\mathbf{L}^{\infty}\left(\mathbb{R}_{+}, \mathbf{L}^{2}\right)}=0
$$

and Theorem 1.1 is proved. 


\section{Appendix A. Contruction of the Ekman layers}

The construction of Ekman boundary layers is classical and can be found in [16] or [9] for instance. In this paper, we will only give very brief recall of this construction. The typical approach consists in looking for the appropriate solutions of the system (1.1) in the following form

$$
\begin{aligned}
& u^{\varepsilon}=u^{I, 0}+u^{B, 0}+\tilde{u}^{B, 0}+\varepsilon\left(u^{I, 1}+u^{B, 1}+\tilde{u}^{B, 1}\right)+\varepsilon^{2}\left(u^{I, 2}+u^{B, 2}+\tilde{u}^{B, 2}\right)+\ldots \\
& p^{\varepsilon}=\frac{1}{\varepsilon}\left(p^{I,-1}+p^{B,-1}+\tilde{p}^{B,-1}\right)+p^{I, 0}+p^{B, 0}+\tilde{p}^{B, 0}+\varepsilon\left(p^{I, 1}+p^{B, 1}+\tilde{p}^{B, 1}\right)+\ldots
\end{aligned}
$$

The "interior part" functions with the index "I" depend on $\left(t, x_{1}, x_{2}, x_{3}\right)$ and the "boundary layer part" with the index "B" consists in smooth functions of the form

$$
\begin{array}{rlrl}
u^{B, j} & =u^{B, j}\left(t, x_{1}, x_{2}, \frac{x_{3}}{\varepsilon}\right), & p^{B, j} & =p^{B, j}\left(t, x_{1}, x_{2}, \frac{x_{3}}{\varepsilon}\right), \\
\tilde{u}^{B, j} & =\tilde{u}^{B, j}\left(t, x_{1}, x_{2}, \frac{1-x_{3}}{\varepsilon}\right), & \tilde{p}^{B, j}=\tilde{p}^{B, j}\left(t, x_{1}, x_{2}, \frac{1-x_{3}}{\varepsilon}\right),
\end{array}
$$

and which rapidly (exponentially) decrease when the third space variable goes to infinity. The justification of the size of the boundary layers being $\varepsilon$ and the discussion about other sizes of the layers can be found in [16], [21], [8] and [9].

A.1. In the interior part of the domain. Away from the boundary, all the boundary terms in (A.1) rapidly decrease to zero. Thus, at the leading order $\varepsilon^{-1}$, the divergence-free conditions and the fast rotation yield

which leads to

$$
u_{2}^{I, 0}=\partial_{1} p^{I,-1}, u_{1}^{I, 0}=-\partial_{2} p^{I,-1}, \partial_{3} p^{I,-1}=0,
$$

$$
\left\{\begin{array}{l}
\partial_{3} u^{I, 0}=0, \partial_{3} p^{I,-1}=0 \\
\partial_{1} u_{1}^{I, 0}+\partial_{2} u_{2}^{I, 0}=0 .
\end{array}\right.
$$

The limiting velocity $u^{I, 0}$ is then a two-dimensional divergence-free vector field with three components, which means that the fluid has tendency to move in columns when the rotation is fast, as predicts the Taylor-Proudman theorem.

Now, looking at the zeroth order $\left(\varepsilon^{0}\right)$, one obtains the governing equation of the fluid at the limit as $\varepsilon \rightarrow 0$

$$
\left\{\begin{array}{l}
\partial_{t} u_{1}^{I, 0}+u_{h}^{I, 0} \cdot \nabla_{h} u_{1}^{I, 0}-u_{2}^{I, 1}+\partial_{1} p^{I, 0}=0 \\
\partial_{t} u_{2}^{I, 0}+u_{h}^{I, 0} \cdot \nabla_{h} u_{2}^{I, 0}+u_{1}^{I, 1}+\partial_{2} p^{I, 0}=0 \\
\partial_{t} u_{3}^{I, 0}+u_{h}^{I, 0} \cdot \nabla_{h} u_{3}^{I, 0}+\partial_{3} p^{I, 0}=0 .
\end{array}\right.
$$

Here, we remark that the fast rotation implies the action of the first order term, i.e. the added vector field $\left(-u_{2}^{I, 1}, u_{1}^{I, 1}, 0\right)$, on the limiting behavior of the fluid. In the next paragraph, we will show that the interaction with the boundary allows to explicitly calculate this vector field, which turns out to be a dissipative term (the Ekman pumping).

A.2. In the Ekman boundary layers. We will focus on the boundary layer near $\left\{x_{3}=0\right\}$. The other layer near $\left\{x_{3}=1\right\}$ can be obtained in a similar way. Performing the change of variable $y=\frac{x_{3}}{\varepsilon}$, we deduce the following "divergence-free properties" of $u^{B, j}$, for any $j \geq 0$,

$$
\left\{\begin{array}{l}
\partial_{y} u_{3}^{B, 0}=0 \\
\partial_{1} u_{1}^{B, j}+\partial_{2} u_{2}^{B, j}+\partial_{y} u_{3}^{B, j+1}=0, \quad \forall j \geq 0 .
\end{array}\right.
$$

We remark that the first equation of (A.4) implies that $u_{3}^{B, 0} \equiv 0$ because it goes to zero as $y \rightarrow+\infty$.

The Dirichlet boundary condition $u^{\varepsilon}\left(t, x_{1}, x_{2}, 0\right)=0$ is rewritten as follows

$$
u^{B, j}\left(t, x_{1}, x_{2}, 0\right)=-u^{I, j}\left(t, x_{1}, x_{2}, 0\right), \quad \forall j \geq 0,
$$

which implies $u_{3}^{I, 0} \equiv 0$, since $u_{3}^{I, 0}$ is independent of $x_{3}$ and $u^{I, 0}\left(t, x_{1}, x_{2}, 0\right)=-u^{B, 0}\left(t, x_{1}, x_{2}, 0\right)=0$.

Now, putting the Ansatz into the first equation of (1.1) and looking at the leading order $\varepsilon^{-2}$, we simply get

$$
\partial_{y} p^{B,-1}=0
$$


and so, $p^{B,-1}=0$ since $p^{B,-1} \rightarrow 0$ as $y \rightarrow+\infty$. This is a classical principle of fluid mechanics which claims that the pressure does not vary in a boundary layer. At the next order $\varepsilon^{-1}$, combining the boundary condition (A.5) and the fact that $u^{I, 0}=u^{I, 0}\left(t, x_{1}, x_{2}\right)$ is independent of $x_{3}$, we recover the following system

$$
\left\{\begin{array}{l}
\beta \partial_{y}^{2} u_{1}^{B, 0}+u_{2}^{B, 0}=0 \\
\beta \partial_{y}^{2} u_{2}^{B, 0}-u_{1}^{B, 0}=0 \\
\left.u_{1}^{B, 0}\right|_{y=0}=-u_{1}^{I, 0},\left.u_{2}^{B, 0}\right|_{y=0}=-u_{2}^{I, 0} \\
\lim _{y \rightarrow+\infty} u_{1}^{B, 0}=\lim _{y \rightarrow+\infty} u_{2}^{B, 0}=0
\end{array}\right.
$$

This system can be explicitly solved and that gives

$$
\left\{\begin{array}{l}
u_{1}^{B, 0}=-e^{-\frac{y}{\sqrt{2 \beta}}}\left(u_{1}^{I, 0} \cos \frac{y}{\sqrt{2 \beta}}+u_{2}^{I, 0} \sin \frac{y}{\sqrt{2 \beta}}\right)=-e^{-\frac{x_{3}}{\varepsilon \sqrt{2 \beta}}}\left(u_{1}^{I, 0} \cos \frac{x_{3}}{\varepsilon \sqrt{2 \beta}}+u_{2}^{I, 0} \sin \frac{x_{3}}{\varepsilon \sqrt{2 \beta}}\right) \\
u_{2}^{B, 0}=-e^{-\frac{y}{\sqrt{2 \beta}}}\left(u_{2}^{I, 0} \cos \frac{y}{\sqrt{2 \beta}}-u_{1}^{I, 0} \sin \frac{y}{\sqrt{2 \beta}}\right)=-e^{-\frac{x_{3}}{\varepsilon \sqrt{2 \beta}}}\left(u_{2}^{I, 0} \cos \frac{x_{3}}{\varepsilon \sqrt{2 \beta}}-u_{1}^{I, 0} \sin \frac{x_{3}}{\varepsilon \sqrt{2 \beta}}\right) .
\end{array}\right.
$$

Using (A.4), (A.7) and the fact that $\partial_{1} u_{1}^{I, 0}+\partial_{2} u_{2}^{I, 0}=0$, we have

$$
\partial_{y} u_{3}^{B, 1}=-\partial_{1} u_{1}^{B, 0}-\partial_{2} u_{2}^{B, 0}=\exp \left(-\frac{y}{\sqrt{2 \beta}}\right) \sin \left(\frac{y}{\sqrt{2 \beta}}\right) \operatorname{curl} u_{h}^{I, 0},
$$

where curl $u_{h}^{I, 0}=\partial_{1} u_{2}^{I, 0}-\partial_{2} u_{1}^{I, 0}$. Integrating the above equation with respect to $y$ and recalling that $u_{3}^{B, 1} \rightarrow 0$ as $y \rightarrow+\infty$, we obtain

$$
u_{3}^{B, 1}=-\sqrt{\beta} e^{-\frac{y}{\sqrt{2 \beta}}} \sin \left(\frac{y}{\sqrt{2 \beta}}+\frac{\pi}{4}\right) \operatorname{curl} u_{h}^{I, 0}=-\sqrt{\beta} e^{-\frac{x_{3}}{\varepsilon \sqrt{2 \beta}}} \sin \left(\frac{x_{3}}{\varepsilon \sqrt{2 \beta}}+\frac{\pi}{4}\right) \operatorname{curl} u_{h}^{I, 0} .
$$

Remark A.1. We remark that unlike the irrotational case where the boundary layers are discribed by the Prandtl equations, in this case, the main boundary layer term $\left(u_{1}^{B, 0}, u_{2}^{B, 0}, u_{3}^{B, 1}\right)$ can be explicitly computed from the limiting velocity in the interior domain, which allows to directly treat these layers without using the Prandtl equations.

The same arguments near the boundary $\left\{x_{3}=1\right\}$ lead to $\tilde{p}^{B,-1}=0$ and

$$
\left\{\begin{array}{l}
\tilde{u}_{1}^{B, 0}=-e^{-\frac{1-x_{3}}{\varepsilon \sqrt{2 \beta}}}\left(u_{1}^{I, 0} \cos \frac{1-x_{3}}{\varepsilon \sqrt{2 \beta}}+u_{2}^{I, 0} \sin \frac{1-x_{3}}{\varepsilon \sqrt{2 \beta}}\right) \\
\tilde{u}_{2}^{B, 0}=-e^{-\frac{1-x_{3}}{\varepsilon \sqrt{2 \beta}}}\left(u_{2}^{I, 0} \cos \frac{1-x_{3}}{\varepsilon \sqrt{2 \beta}}-u_{1}^{I, 0} \sin \frac{1-x_{3}}{\varepsilon \sqrt{2 \beta}}\right) \\
\tilde{u}_{3}^{B, 1}=\sqrt{\beta} e^{-\frac{1-x_{3}}{\varepsilon \sqrt{2 \beta}}} \sin \left(\frac{1-x_{3}}{\varepsilon \sqrt{2 \beta}}+\frac{\pi}{4}\right) \operatorname{curl} u_{h}^{I, 0}
\end{array}\right.
$$

We come back to (A.3). Taking $\operatorname{curl} u_{h}^{I, 0}=\partial_{1} u_{2}^{I, 0}-\partial_{2} u_{1}^{I, 0}$, we have

$$
\partial_{t} \operatorname{curl} u_{h}^{I, 0}+u_{h}^{I, 0} \cdot \nabla_{h} \operatorname{curl} u_{h}^{I, 0}=-\partial_{1} u_{1}^{I, 1}-\partial_{2} u_{2}^{I, 1}=\partial_{3} u_{3}^{I, 1} .
$$

Since $u^{I, 0}$ is independent of $x_{3}$, by integrating (A.10) with respect to $x_{3}$ over [0,1], one gets

$$
\partial_{t} \operatorname{curl} u_{h}^{I, 0}+u_{h}^{I, 0} \cdot \nabla_{h} \operatorname{curl} u_{h}^{I, 0}=u_{3}^{I, 1}{ }_{\left.\right|_{x_{3}=1}}-u_{3}^{I, 1}{ }_{\left.\right|_{x_{3}=0}}=u_{3}^{B, 1}{ }_{\left.\right|_{x_{3}=0}}-\tilde{u}_{3}^{B, 1}{ }_{\left.\right|_{x_{3}=1}}=\sqrt{2 \beta} \operatorname{curl} u_{h}^{I, 0} .
$$

Using the Biot-Savart law, we can finally rewrite the system (A.3) in the same form as (1.3)

$$
\left\{\begin{array}{l}
\partial_{t} u_{1}^{I, 0}+u_{h}^{I, 0} \cdot \nabla_{h} u_{1}^{I, 0}+\sqrt{2 \beta} u_{1}^{I, 0}+\partial_{1} p^{I, 0}=0 \\
\partial_{t} u_{2}^{I, 0}+u_{h}^{I, 0} \cdot \nabla_{h} u_{2}^{I, 0}+\sqrt{2 \beta} u_{2}^{I, 0}+\partial_{2} p^{I, 0}=0 \\
\partial_{3} u^{I, 0} \equiv 0, u_{3}^{I, 0} \equiv 0, \\
\partial_{1} u_{1}^{I, 0}+\partial_{2} u_{2}^{I, 0}=0 .
\end{array}\right.
$$




\section{REFERENCES}

[1] A. Babin, A. Mahalov and B. Nicolaenko, Global Splitting, Integrability and Regularity of 3D Euler and Navier-Stokes Equations for Uniformly Rotating Fluids, European Journal of Mechanics, 15, 1996, 291-300.

[2] A. Babin, A. Mahalov and B. Nicolaenko, Global regularity of 3D rotating Navier-Stokes equations for resonant domains, Indiana University Mathematics Journal, 48, 1999, 1133-1176.

[3] H. Bahouri, J.-Y. Chemin, Équations de transport relatives à des champs de vecteurs non-lipschitziens et mécanique des fluides [Transport equations for non-Lipschitz vector fields and fluid mechanics], Arch. Rational Mech. Anal., 127, 1994, no. 2, 159-181.

[4] J.-M. Bony, Calcul symbolique et propagation des singularités pour les équations aux dérivées partielles non linéaires, Annales de l'École Normale Supérieure, 14, 1981, 209-246.

[5] J.-Y. Chemin, Fluides parfaits incompressibles, Astérisque, 230, 1995.

[6] J.-Y. Chemin, B. Desjardins, I. Gallagher and E. Grenier, Anisotropy and dispersion in rotating fluids, Nonlinear Partial Differential Equations and their application, Collège de France Seminar, Studies in Mathematics and its Applications, 31, 2002, 171-191.

[7] J.-Y. Chemin, B. Desjardins, I. Gallagher and E. Grenier, Fluids with anisotropic viscosity, Special issue for R. Temam's 60th birthday, M2AN. Mathematical Modelling and Numerical Analysis, 34, no. 2, 2000, 315-335.

[8] J.-Y. Chemin, B. Desjardins, I. Gallagher, E. Grenier, Ekman boundary layers in rotating fluids, ESAIM Controle optimal et calcul des variations, A tribute to J.-L. Lions, 8, 2002, 441-466.

[9] J.-Y. Chemin, B. Desjardins, I. Gallagher and E. Grenier, Mathematical Geophysics: An introduction to rotating fluids and to the Navier-Stokes equations, Oxford University Press, 2006.

[10] J.-Y. Chemin and N. Lerner, Flot de champs de vecteurs non Lipschitziens et équations de Navier-Stokes, Journal of Differential Equations, 121, 1992, 314-328.

[11] B. Cushman-Roisin, Introduction to geophysical fluid dynamics, Prentice-Hall, 1994.

[12] H. Fujita and T. Kato, On the Navier-Stokes initial value problem I, Archiv for Rational Mechanic Analysis, 16, 1964, 269-315.

[13] I. Gallagher, Applications of Schochet's Methods to Parabolic Equation, Journal de Mathématiques Pures et Appliquées, 77, 1998, 989-1054.

[14] I. Gallagher and L. Saint-Raymond, Weak convergence results for inhomogeneous rotating fluid equations, Journal d'Analyse Mathématique, 99, 2006, 1-34.

[15] H.P. Greenspan, The theory of rotating fluids, Cambrigde University Press, 1968.

[16] E. Grenier, N. Masmoudi, Ekman layers of rotating fluid, the case of well prepared initial data, Communications in Partial Differential Equations, 22 (5-6), 1997, 953-975.

[17] D. Iftimie, The resolution of the Navier-Stokes equations in anisotropic spaces, Revista Matemática Iberoamericana, 15, 1999, 1-36.

[18] A. Kiselev, V. Šverák, Small scale creation for solutions of the incompressible two-dimensional Euler equation, Ann. of Math. (2), 180, 2014, no. 3, 1205-1220.

[19] J. Leray, Essai sur le mouvement d'un liquide visqueux emplissant l'espace, Acta Matematica, 63, 1933, 193-248.

[20] N. Masmoudi, The Euler Limit of the Navier-Stokes Equations, and Rotating Fluids with Boundary, Archive for Rational Mechanics and Analysis, 142, 1998, no. 4, 375-394.

[21] N. Masmoudi, Ekman layers of rotating fluids: the case of general initial data, Communications on Pure and Applied Mathematics, 53, 2000, no. 4, 432-483.

[22] V-S. Ngo, Rotating Fluids with small viscosity, International Mathematics Research Notices IMRN, 2009, no. 10, 1860-1890

[23] M. Paicu, Étude asymptotique pour les fluides anisotropes en rotation rapide dans le cas périodique, Journal de Mathématiques Pures et Appliquées, 83, no. 2, 2004, 163-242.

[24] M. Paicu, Équation anisotrope de Navier-Stokes dans des espaces critiques, Revista Matemática Iberoamericana, 21, 2005, no. 1, 179-235.

[25] J. Pedlosky, Geophysical fluid dynamics, Springer, 1979.

[26] W.J. Schmitz, Weakly depth-dependent segments of the North Atlantic circulation, Journal of Marine Research, 38 , 111-133.

[27] S. Schochet, Fast singular limits of hyperbolic PDEs, Journal of Differential Equations, 114, 1994, 476-512.

[28] V. Yudovitch, Non stationnary flows of an ideal incompressible fluid, Zh. Vych. Math., 3, 1963, 1032-1066.

Laboratoire de Mathématiques Raphä̈l Salem UMR 6085 CNRS, Université De Rouen, 76801 Saint-Etienne du Rouvray Cedex, France.

E-mail address: van-sang.ngo@univ-rouen.fr 